\title{
SYMBOLIC BLENDER-HORSESHOES AND APPLICATIONS
}

\author{
PABLO G. BARRIENTOS, YURI KI AND ARTEM RAIBEKAS
}

\begin{abstract}
Aвstract. We study partially-hyperbolic skew-product maps over the Bernoulli shift with Hölder dependence on the base points. In the case of contracting fiber maps, symbolic blender-horseshoe is defined as an invariant set which meets any almost horizontal disk in a robust sense. These invariant sets are understood as blenders with center stable bundle of any dimension. We then give necessary conditions (covering property) on an iterated function system such that the relevant skew-product has a symbolic blender-horseshoe. We use this local plug to yield robustly non-hyperbolic transitive diffeomorphisms and robust heterodimensional cycles of co-index equal to the dimension of the central direction.
\end{abstract}

\section{INTRODUCTION}

Currently hyperbolic systems are quite well understood from the topological and statistical perspectives, see [BDV05]. A natural objective is to characterize obstructions to hyperbolicity. In [Pa100], Palis conjectured that generation of cycles (heterodimensional cycles and homoclinic tangencies) is the only obstruction: every diffeomorphism can be approximated either by a hyperbolic one or by one with a cycle. This conjecture was proved for surface $C^{1}$-diffeomorphisms in [PS00] and in higher dimensions there are some partial results, see [CP10].

On the other hand, dynamical properties which exhibit some "persistence" play an important role in dynamics. A property of a diffeomorphism is robust if there is a neighborhood consisting of diffeomorphisms satisfying such a property. Bearing in mind this concept, Bonatti formulated in [Bon04] a strong version of "hyperbolicity versus cycles" conjecture: the set of hyperbolic diffeomorphisms and the set of diffeomorphisms with robust cycles are two open sets whose union is dense in the space of $C^{1}$-diffeomorphisms. This conjecture was proved in [BD08] for the so-called "tame systems".

In this paper we study the generation of $C^{1}$-robust cycles continuing the work of [BD08, BD12]. One of the main tools used are the so-called "blenders", which are hyperbolic sets in some sense similar to the thick horseshoes introduced by Newhouse [New70] in the setting of homoclinic tangencies. Roughly, a blender is a local plug that guarantees the closure of an invariant manifold of a hyperbolic set to be greater than the dimension of its unstable bundle.

1991 Mathematics Subject Classification. Primary: 58F15, 58F17; Secondary: 53C35.

Key words and phrases. symbolic skew-products, one-step maps, iteration function systems, symbolic blenders, heterodimensional cycles, robust transitivity. 
Besides playing a key role in the generation of robust cycles (see [BD08, BD12]), blenders are also important in other settings such as construction of non-hyperbolic robustly transitive sets [BD96], discontinuity of the dimension of hyperbolic sets [BDV95], stable ergodicity [RHRHTU07], and Arnold difusion in symplectic and Hamiltonian settings [NP12, FM01].

The papers [BD96, BD08, BD12] consider blenders having one-dimensional "central" direction, see Definition 1.3. Here, following the ideas in [NP12] we study blenders with "central" direction of any dimension, which enables us to consider these sets in more general settings, and as applications we get the next results.

1.1. Robust heterodimensional cycles and mixing sets. Let $M$ be a manifold and consider $\operatorname{Diff}^{1}(M)$ the set of diffeomorphisms $f: M \rightarrow M$ endowed with the $C^{1}$-topology. A heterodimensional cycle for $f \in \operatorname{Diff}^{1}(M)$ between a pair of hyperbolic transitive sets $\Omega_{1}$ and $\Omega_{2}$ of different indices (dimension of their stable bundles) is defined by the relations

$$
W^{s}\left(\Omega_{1}, f\right) \cap W^{u}\left(\Omega_{2}, f\right) \neq \emptyset \quad \text { and } \quad W^{u}\left(\Omega_{1}, f\right) \cap W^{s}\left(\Omega_{2}, f\right) \neq \emptyset .
$$

The co-index is the absolute value of the difference between the indices of $\Omega_{1}, \Omega_{2}$. We are interested in cycles that cannot be destroyed by perturbations.

Definition 1.1 (Robust heterodimensional cycle). A diffeomorphism $f$ has a $C^{1}$-robust heterodimensional cycle associated with its transitive hyperbolic sets $\Omega_{1}$ and $\Omega_{2}$ if there is a $C^{1}$-neighborhood $\mathcal{U}$ of $f$ such that every $g \in \mathcal{U}$ has a heterodimensional cycle associated with the hyperbolic continuations $\Omega_{1}^{g}$ and $\Omega_{2}^{g}$ of $\Omega_{1}$ and $\Omega_{2}$, respectively.

In [BD08] it is proved that cycles of co-index one yield $C^{1}$-robust cycles after small perturbations. A key ingredient is the construction of blenders with one dimensional center-stable direction. Here we consider $F: N \rightarrow N$ a $C^{1}$-diffeomorphism with a horseshoe, $M$ a manifold of dimension $c \geq 1$ and id the identity map. We prove that there are maps arbitrarily close to $F \times$ id having robust cycles. The following result is a generalization of [BD08, Theorem 2.4].

Theorem A. Let $F: N \rightarrow N$ be a $C^{1}$-diffeomorphism with a Smale horseshoe. Then there is an $\operatorname{arc}\left\{f_{\varepsilon}\right\}_{\varepsilon \in\left(0, \varepsilon_{0}\right]}$ of $C^{1}$-diffeomorphisms on $N \times M$ such that $f_{0}=F \times$ id and $f_{\varepsilon}$ has a $C^{1}$-robust heterodimensional cycle of co-index c for all $0<\varepsilon<\varepsilon_{0}$.

The construction in [BD96] of persistent non-hyperbolic transitive diffeomorphisms used a "chain of blenders", which is a connected family of blenders. Here, we provide an alternative construction using symbolic blenders (the precise definition will be given in Section 1.3), thus obtaining a slightly stronger, topologically mixing, version of the theorem. An invariant set $X$ is topologically mixing for a map $g$ if for any pair of open sets $U, V$ of $X$ there is a $n_{0}>0$ such that $g^{n}(U) \cap V \neq \emptyset$ for all $n \geq n_{0}$.

Theorem B. Let $F: N \rightarrow N$ be a $C^{1}$-diffeomorphism with a Smale horseshoe $\Lambda$. Then there is an $\operatorname{arc}\left\{f_{\varepsilon}\right\}_{\varepsilon \in\left(0, \varepsilon_{0}\right]}$ of $C^{1}$-diffeomorphisms on $N \times M$ such that $f_{0}=F \times$ id and for every small enough $C^{1}$-perturbation $g$ of $f_{\varepsilon}, 0<\varepsilon<\varepsilon_{0}$, there is a g-invariant set $\Delta_{g} \subset N \times M$ homeomorphic to $\Lambda \times M$ which is a non-hyperbolic topologically mixing set for $g$. 
1.2. The set of symbolic skew-products. Blenders appear in a natural way in the context of skew-product diffeomorphisms

$$
f:[-1,1]^{n} \times[-1,1] \rightarrow \mathbb{R}^{n} \times \mathbb{R}, \quad f(x, y)=(F(x), \phi(x, y)),
$$

where $n \geq 2, F: \mathbb{R}^{n} \rightarrow \mathbb{R}^{n}$ has a horseshoe $\Lambda \subset[-1,1]^{n}$, and the fiber maps $\phi(x, \cdot): \mathbb{R} \rightarrow \mathbb{R}$ are contractions. Since $\left.F\right|_{\Lambda}$ is conjugate to a shift, this map can be studied symbolically. This leads to consider symbolic skew-product maps $\Phi$ over the shift map $\tau: \Sigma_{k} \stackrel{\text { def }}{=}\{1, \ldots, k\}^{\mathbb{Z}} \rightarrow \Sigma_{k}$ of $k \geq 2$ symbols of the form

$$
\Phi: \Sigma_{k} \times M \rightarrow \Sigma_{k} \times M, \quad \Phi(\xi, x)=\left(\tau(\xi), \phi_{\xi}(x)\right),
$$

where $M$ is a manifold and $\phi_{\xi}: M \rightarrow M$ are diffeomorphisms depending continuously with respect to $\xi$. The set $\Sigma_{k}$ is called base and $M$ is the fiber. In this paper we will work with maps of the form (1.1) and in order to emphasize the role of the fiber maps we write $\Phi=\tau \ltimes \phi_{\xi}$.

Let $D$ be a bounded open set of $M$. Given positive constants $0<\lambda<\beta$, a map $\phi: \bar{D} \rightarrow D$ is said to be a $(\lambda, \beta)$-Lipschitz on $\bar{D}$ if

$$
\lambda\|x-y\|<\|\phi(x)-\phi(y)\|<\beta\|x-y\|, \quad \text { for all } x, y \in \bar{D},
$$

where $\|x-y\|$ denotes the distance between $x$ and $y$ in $M$. Fix $0<v<1$ and the metric

$$
d_{\Sigma_{k}}(\xi, \zeta) \stackrel{\text { def }}{=} v^{\ell}, \quad \ell=\min \left\{i \in \mathbb{Z}^{+}: \xi_{i} \neq \zeta_{i} \text { or } \xi_{-i} \neq \zeta_{-i}\right\} .
$$

For a fixed $0 \leq \alpha \leq 1$, we say that $\Phi=\tau \ltimes \phi_{\xi}$ is locally $\alpha$-Hölder continuous (or that its fiber maps $\phi_{\xi}$ depend locally $\alpha$-Hölder continuously on $\bar{D}$ with respect to the base point $\xi$ ) if there is a constant $C \geq 0$ such that

$$
\left\|\phi_{\xi}^{ \pm 1}(x)-\phi_{\zeta}^{ \pm 1}(x)\right\| \leq C d_{\Sigma_{k}}(\xi, \zeta)^{\alpha}, \quad \text { for all } x \in \bar{D}
$$

and every $\xi, \zeta \in \Sigma_{k}$ with $\xi_{0}=\zeta_{0}$. We denote by $C_{\Phi}$ the smallest non-negative constant satisfying (1.2) and call it the local Hölder constant of $\Phi$ on $\bar{D}$.

Definition 1.2 (Sets of symbolic skew-products). Let $D \subset M$ be a bounded open set, $r \geq 0$, $0<\lambda<\beta, 0 \leq \alpha \leq 1$ and $k>1$. We define $\mathcal{S}_{k, \lambda, \beta}^{r, \alpha}(D)$ as the set of symbolic skew-product maps $\Phi=\tau \ltimes \phi_{\xi}$ as in (1.1) such that

- $\phi_{\xi}$ is $C^{r}-(\lambda, \beta)$-Lipschitz on $\bar{D}$ for all $\xi \in \Sigma_{k}$,

- $\phi_{\xi}$ depends locally $\alpha$-Hölder continuously on $\bar{D}$ with respect to $\xi$.

The set $\mathcal{S}_{k, \lambda, \beta}^{r, \alpha}(D)$ is endowed with the distance

$$
d_{\mathcal{S}}(\Phi, \Psi)=\sup _{\xi \in \Sigma_{k}} d_{C^{r}}\left(\phi_{\xi}, \psi_{\xi}\right)+\left|C_{\Phi}-C_{\Psi}\right|,
$$

where $\Phi=\tau \ltimes \phi_{\xi}$ and $\Psi=\tau \ltimes \psi_{\xi}$.

Since we will be working with hyperbolic invariant sets generated by contracting or expanding fiber maps, we will always assume the following hypothesis. In the case $\beta<1$ (contracting fiber maps) then $\phi_{\xi}(\bar{D}) \subset D$ for all $\xi \in \Sigma_{k}$, and if $1<\lambda$ (expanding fiber maps) then $\bar{D} \subset \phi_{\xi}(D)$ for all $\xi \in \Sigma_{k}$. 
1.3. Symbolic blender-horseshoes. Before introducing our main tool symbolic blenderhorseshoes let us recall the definition of a blender (with one-dimensional center contracting direction) in [BDV05, BD12]:

Definition 1.3 (cs-blenders). Let $f$ be a $C^{1}$-diffeomorphism of manifold $M$ and $\Gamma \subset M$ a transitive hyperbolic set of $f$ with a dominated splitting of the form $E^{s S} \oplus E^{c} \oplus E^{u u}$, where its stable bundle $E^{s}=E^{s s} \oplus E^{c}$ has dimension equal to $k \geq 2$ and $E^{c}$ is one-dimensional. The set $\Gamma$ is a cs-blender if it has a $C^{1}$-robust superposition region of embedding disks, that is, there are a $C^{1}$-neighborhood $\mathcal{V}$ of $f$ and $a C^{1}$-open set $\mathcal{H}$ of embeddings of $k-1$ dimensional disks into $M$ such that for every diffeomorphism $g \in \mathcal{V}$, every disk $H \in \mathcal{H}$ intersects the local (strong) unstable manifold $W_{\text {loc }}^{u u}\left(\Gamma_{g}\right)$ of the continuation $\Gamma_{g}$ of $\Gamma$ for $g$.

To adapt the definition of a blender to the symbolic context we need to define the family of almost horizontal disks, which provides the superposition region.

Definition 1.4 (Almost horizontal disks). Given $\delta>0,0 \leq \alpha \leq 1$ and a bounded open set $B \subset M$, we say that a set $H^{s} \subset \Sigma_{k} \times M$ is an almost $\delta$-horizontal disk in $\Sigma_{k} \times B$ if there are $(\zeta, z) \in \Sigma_{k} \times B$ and $a(\alpha, C)$-Hölder function

$$
h: W_{l o c}^{s}(\zeta ; \tau) \rightarrow \text { B such that }\|z-h(\xi)\|<\delta \text { for all } \xi \in W_{l o c}^{s}(\zeta ; \tau),
$$

with

$$
C v^{\alpha}<\delta \quad \text { and } \quad H^{s}=\left\{(\xi, h(\xi)): \xi \in W_{l o c}^{s}(\zeta ; \tau)\right\}
$$

Here $W_{l o c}^{s}(\zeta ; \tau) \stackrel{\text { def }}{=}\left\{\xi \in \Sigma_{k}: \xi_{i}=\zeta_{i}, i \geq 0\right\}$ is the local stable set of $\zeta$ for $\tau$.

In the case of an invariant hyperbolic set of a diffeomorphism, it is known the existence of local (strong) unstable manifolds. An analogous result holds for the maximal invariant set $\Gamma$ in $\Sigma_{k} \times \bar{D}$ of a symbolic skew-product $\Phi$ in $\mathcal{S}_{k, \lambda, \beta}^{r, \alpha}(D)$ with $\beta<1$ and $\alpha>0$ (more details in Section 2). We denote by $W_{l o c}^{u u}((\xi, x) ; \Phi)$ these local strong unstable sets and define the local strong unstable set of $\Gamma$ as

$$
W_{l o c}^{u u}(\Gamma ; \Phi)=\bigcup_{x \in \Gamma} W_{l o c}^{u u}((\xi, x) ; \Phi) .
$$

We are now ready to formulate the definition of a symbolic blender.

Definition 1.5 (Symbolic cs-blender-horseshoe). Consider a symbolic skew-product map $\Phi \in$ $\mathcal{S}_{k, \lambda, \beta}^{0, \alpha}(D)$ with $\beta<1$ and $\alpha>0$. The maximal invariant set $\Gamma_{\Phi}$ of $\Phi$ in $\Sigma_{k} \times \bar{D}$ is a symbolic cs-blender-horseshoe if there are $\delta>0$, a non-empty open set $B \subset D$, and a neighborhood $\mathcal{V}$ of $\Phi$ in $\mathcal{S}_{k, \lambda, \beta}^{0, \alpha}(D)$ such that for every $\Psi \in \mathcal{V}$ and every $\delta$-horizontal disk $H^{s}$ in $\Sigma_{k} \times B$ it holds that

$$
W_{l o c}^{u u}\left(\Gamma_{\Psi} ; \Psi\right) \cap H^{s} \neq \emptyset,
$$

where $\Gamma_{\Psi}$ is the maximal invariant set of $\Psi$ in $\Sigma_{k} \times \bar{D}$.

The family of $\delta$-horizontal disks in $\Sigma_{k} \times B$ is called the superposition region of the symbolic blenders and the open set $B$ is the superposition domain. 
A special case of symbolic skew-product maps are the one-step ones where the fiber maps $\phi_{\xi}$ only depend on the coordinate $\xi_{0}$ of $\xi=\left(\xi_{i}\right)_{i \in \mathbb{Z}} \in \Sigma_{k}$. So, we have that $\phi_{\xi}=\phi_{i}$ if $\xi_{0}=i$, and write $\Phi=\tau \ltimes\left(\phi_{1}, \ldots, \phi_{k}\right)$. Symbolic blenders in the one-step setting were first introduced in [NP12]. In this paper, blender-horseshoes are obtained by small perturbations in $\mathcal{S}_{k, \lambda, \beta}^{0, \alpha}(D)$ of one-step skew-product maps.

Next result gives conditions for the existence of blenders.

Theorem C. Consider $\Phi=\tau \ltimes\left(\phi_{1}, \ldots, \phi_{k}\right) \in \mathcal{S}_{k, \lambda, \beta}^{0, \alpha}(D)$ with $\alpha>0$ and $v^{\alpha}<\lambda<\beta<1$. Assume that there exists an open set $B \subset D$ such that

$$
\bar{B} \subset \phi_{1}(B) \cup \cdots \cup \phi_{k}(B) .
$$

Then the maximal invariant set $\Gamma_{\Phi}$ of $\Phi$ in $\Sigma_{k} \times \bar{D}$ is a symbolic cs-blender-horseshoe for $\Phi$ whose superposition domain contains $B$.

1.4. Standing notation. Given a bi-sequence $\xi=\left(\ldots, \xi_{-1} ; \xi_{0}, \xi_{1}, \ldots\right) \in \Sigma_{k}$ the symbol at the right of ";" is the " 0 coordinate" of $\xi$. We define the local stable/unstable set of $\xi$ for $\tau$ as

$$
W_{l o c}^{s}(\xi ; \tau)=\left\{\zeta \in \Sigma_{k}: \zeta_{i}=\xi_{i}, i \geq 0\right\} \quad \text { and } \quad W_{l o c}^{u}(\xi ; \tau)=\left\{\zeta \in \Sigma_{k}: \zeta_{i}=\xi_{i}, i \leq 0\right\} .
$$

Given $\Phi=\tau \ltimes \phi_{\xi}$, for every $n>0$ and every $(\xi, x) \in \Sigma_{k} \times \bar{D}$ we set

$$
\begin{aligned}
\phi_{\xi}^{n}(x) & \stackrel{\text { def }}{=} \phi_{\tau^{n-1}(\xi)} \circ \cdots \circ \phi_{\xi}(x), \\
\phi_{\xi}^{-n}(x) & \stackrel{\text { def }}{=} \phi_{\tau^{-(n-1)}(\xi)}^{-1} \circ \cdots \circ \phi_{\xi}^{-1}(x) .
\end{aligned}
$$

Note that for every $n \geq 0$,

$$
\Phi^{n}(\xi, x)=\left(\tau^{n}(\xi), \phi_{\xi}^{n}(x)\right) \text { and } \Phi^{-n}(\xi, x)=\left(\tau^{-n}(\xi), \phi_{\tau^{-1}(\xi)}^{-n}(x)\right) .
$$

For simplicity we write $\mathcal{S}$ in the place of $\mathcal{S}_{k, \lambda, \beta}^{0, \alpha}(D)$, where the set $D \subset M$ and all the constants, $k \in \mathbb{N}, 0<\lambda<\beta, \alpha \geq 0$, are fixed.

1.5. Organization of the paper. In the next section we will study symbolic skew-products in $\mathcal{S}$, and properties of the maximal invariant set in $\Sigma_{k} \times \bar{D}$. In Section 3 we will look at symbolic blender-horseshoes in the one-step setting and then prove Theorem $C$ in Section 4. Finally, in Section 5, we will prove Theorems A and B. In Appendix A we include the reduction to perturbation of symbolic skew-products.

\section{SYMBOLIC SKEW-PRODUCTS}

In this section given $\Phi=\tau \ltimes \phi_{\xi} \in \mathcal{S}$ with $\alpha>0$, we will first study the existence of strong stable and unstable laminations. Afterwards, we will focus on skew-products with uniformly locally contracting fiber maps and analyze the properties of the maximal invariant set. 
2.1. Global strong stable and unstable laminations. For this section let the domain $D$ of the fiber maps of $\Phi$ be the whole manifold $M$, and let us assume the global domination condition $v^{\alpha}<\lambda<\beta<v^{-\alpha}$. Then the familiar graph transform argument yields the local strong stable and unstable laminations for the symbolic skew-product. To this end, we define the local stable set of $(\xi, x)$ for $\Phi$ as

$$
W^{s}((\xi, x) ; \Phi) \stackrel{\text { def }}{=}\left\{(\zeta, y) \in \Sigma_{k} \times M: \lim _{n \rightarrow \infty} d\left(\Phi^{n}(\zeta, y), \Phi^{n}(\xi, x)\right)=0\right\}
$$

Proposition 2.1 ([AV10]). Let $\Phi \in \mathcal{S}_{k, \lambda, \beta}^{0, \alpha}(M)$ be a symbolic skew-product satisfying the s-domination condition $v^{\alpha}<\lambda$. Then, there exists a partition

$$
\mathcal{W}_{\Phi}^{s s}=\left\{W_{l o c}^{s s}((\xi, x) ; \Phi):(\xi, x) \in \Sigma_{k} \times M\right\}
$$

of $\Sigma_{k} \times M$ such that denoting $C=C_{\Phi}\left(1-\lambda^{-1} v^{\alpha}\right)^{-1} \geq 0$, it holds

i) every leaf $W_{l o c}^{s s}((\xi, x) ; \Phi)$ is the graph of an $\alpha$-Hölder function

$$
\gamma_{\xi, x, \Phi}^{s}: W_{l o c}^{s}(\xi ; \tau) \rightarrow M
$$

with $\alpha$-Hölder constant less or equal than $C$ (uniform on $\xi$ and $x$ ), and it depends continuously on $\Phi$,

ii) $W_{l o c}^{\text {ss }}((\xi, x) ; \Phi)$ varies continuously with respect to $(\xi, x)$, i.e, the map

$$
\left(\xi, \xi^{\prime}, x\right) \mapsto \gamma_{\xi, x, \Phi}^{s}\left(\xi^{\prime}\right) \quad \text { is continuous }
$$

where $\left(\xi, \xi^{\prime}\right)$ varies in the space of pairs of points in the same local stable set for $\tau$,

iii) $\Phi\left(W_{l o c}^{s s}((\xi, x) ; \Phi)\right) \subset W_{l o c}^{s s}(\Phi(\xi, x) ; \Phi)$ for all $(\xi, x) \in \Sigma_{k} \times M$,

iv) $W_{\text {loc }}^{s s}((\xi, x) ; \Phi) \subset W^{s}((\xi, x) ; \Phi)$.

There is a dual statement of Proposition 2.1 for the symbolic skew-product $\Phi$ satisfying the $u$-dominating condition $\beta<v^{-\alpha}$. Namely, there exists a partition $\mathcal{W}_{\Phi}^{u u}=\left\{W_{l o c}^{u u}((\xi, x) ; \Phi)\right.$ : $\left.(\xi, x) \in \Sigma_{k} \times M\right\}$ of $\Sigma_{k} \times M$ verifying analogous properties. Each leaf $W_{l o c}^{u u}((\xi, x) ; \Phi)$ of this partition $\mathcal{W}_{\Phi}^{u}$ is said to be local strong unstable set throughout the point $(\xi, x)$. We define the global strong unstable set of $(\xi, x)$ as

$$
W^{u u}((\xi, x) ; \Phi) \stackrel{\text { def }}{=} \bigcup_{n \geq 0} \Phi^{n}\left(W_{l o c}^{u u}\left(\Phi^{-n}(\xi, x) ; \Phi\right)\right) \subset W^{u}((\xi, x) ; \Phi) .
$$

For a $\Phi$-invariant set $\Gamma$ we define local strong unstable set of $\Gamma$ for $\Phi$ as

$$
W_{l o c}^{u u}(\Gamma ; \Phi)=\bigcup_{(\xi, x) \in \Gamma} W_{l o c}^{u u}((\xi, x) ; \Phi) .
$$

and the global unstable set of $\Gamma$ for $\Phi$ as

$$
W^{u}(\Gamma ; \Phi) \stackrel{\text { def }}{=}\left\{(\xi, x) \in \Sigma_{k} \times M: \lim _{n \rightarrow \infty} d\left(\Phi^{-n}(\xi, x), \Gamma_{\Phi}\right)=0\right\} .
$$

In the same manner, the global strong unstable set of $\Gamma$, denoted by $W^{u u}(\Gamma ; \Phi)$, is defined as the union of the global strong unstable sets of the points in $\Gamma$ and it holds

$$
W_{\text {loc }}^{u u}(\Gamma ; \Phi) \subset W^{u u}(\Gamma ; \Phi) \subset W^{u}(\Gamma ; \Phi) .
$$

Similarly, local/global (strong) stable sets are defined and satisfy analogous inclusions. 
Let us call such skew-products with the global domination property as partially hyperbolic. In part this is motivated by the reduction of partially hyperbolic diffeomorphisms to the setting of symbolic skew-products discussed in the Appendix A.

Definition 2.2 (Partially hyperbolic symbolic skew-products). A symbolic skew-product $\Phi \in$ $\mathcal{S}_{k, \lambda, \beta}^{r, \alpha}(M)$ is called partially hyperbolic if $v^{\alpha}<\lambda<1<\beta<v^{-\alpha}$. We will denote by $\mathcal{P} \mathcal{H} \mathcal{S}_{k}^{r, \alpha}(M)$ the set of partially hyperbolic symbolic $\alpha$-Hölder skew-products on $\Sigma_{k} \times M$ with $C^{r}$-fiber maps.

2.2. Skew-products with locally contracting fibers. Suppose now that the fiber maps $\phi_{\xi}$ of the skew-product $\Phi=\tau \ltimes \phi_{\xi} \in \mathcal{S}$ are locally contracting on the open set $D \subset M$, that is $\beta<1$ and $D$ is forward invariant by $\phi_{\xi}$. These are the hypothesis in the definition of the symbolic blender. We will analyze the dynamics of the maximal invariant set $\Gamma_{\Phi}$ in $\Sigma_{k} \times \bar{D}$.

We will also need a local version of Proposition 2.1 from the previous section on the existence of the unstable partition when there is a global $u$-domination. The same principles used in that proof can be applied to show the existence of a partition $\mathcal{W}_{\Gamma_{\Phi}}^{u u}$ of $\Gamma_{\Phi}$ such that every leaf $W_{l o c}^{u u}((\xi, x) ; \Phi),(\xi, x) \in \Gamma_{\Phi}$, is the graph of an $\alpha$-Hölder map $\gamma_{\xi, x, \Phi}^{u}: W_{l o c}^{u}(\xi ; \tau) \rightarrow M$, and

$$
W_{l o c}^{u u}((\xi, x) ; \Phi) \subset W^{u}((\xi, x) ; \Phi) .
$$

In fact, $\Gamma_{\Phi}$ is an invariant attracting graph for $\Phi$ and, thus, $\left.\Phi\right|_{\Gamma_{\Phi}}$ is conjugate to $\left.\tau\right|_{\Sigma_{k}}$ and the projection of $\Gamma_{\Phi}$ in $M$ depends continuously in the Hausdorff metric on $\Phi$. These are a version of well-known results from [HPS77] in our setting of symbolic skew-products. We summarize these properties of the maximal invariant set in the following theorem and for the completeness of the paper, we give, in our symbolic setting, its non-trivial proof in Appendix B. Before stating, we denote by $\operatorname{Per}(\Phi)$ the set of periodic points of $\Phi$ and that $\mathscr{P}: \Sigma_{k} \times M \rightarrow M$ is the projection on the fiber space.

Theorem 2.3. Consider $\Phi \in \mathcal{S}_{k, \lambda, \beta}^{0, \alpha}(D)$ with $\beta<1$ and $\alpha>0$. Then

$$
\Gamma_{\Phi} \stackrel{\text { def }}{=} \bigcap_{n \in \mathbb{Z}} \Phi^{n}\left(\Sigma_{k} \times \bar{D}\right)=\bigcap_{n \in \mathbb{N}} \Phi^{n}\left(\Sigma_{k} \times \bar{D}\right) .
$$

Moreover, the following holds:

i) the restriction of $\Phi$ to $\Gamma_{\Phi}$ is conjugate to the full shift $\tau$ of $k$ symbols,

ii) $W^{u u}((\xi, x) ; \Phi)=W^{u}((\xi, x) ; \Phi) \subset \Gamma_{\Phi}$ for all $(\xi, x) \in \Gamma_{\Phi}$,

iii) there exists a unique continuous function $g_{\Phi}: \Sigma_{k} \rightarrow \bar{D}$ such that for every periodic point $(\vartheta, p)$ of $\Phi$ it holds,

$$
\mathscr{P}\left(\Gamma_{\Phi}\right)=\overline{\operatorname{Per}(\Phi) \cap D} \stackrel{\text { def }}{=} K_{\Phi} \in \mathcal{K}(D)
$$

and

$$
\Gamma_{\Phi}=W_{l o c}^{u u}\left(\Gamma_{\Phi} ; \Phi\right)=\overline{\left.W^{u}((\vartheta, p) ; \Phi)\right)}=\left\{\left(\xi, g_{\Phi}(\xi)\right): \xi \in \Sigma_{k}\right\},
$$

iv) the map $\mathscr{L}: \mathcal{S}_{k, \lambda, \beta}^{0, \alpha}(D) \rightarrow \mathcal{K}(\bar{D})$ given by $\mathscr{L}(\Phi)=K_{\Phi}$ is continuous. 
3. Symbolic BLENDERS IN THE ONE-STEP SETTING

Let us introduce the definition given in [NP12] for one-step symbolic blenders. First consider the subset $Q=Q_{k, \lambda, \beta}^{0}(D)$ of $\mathcal{S}=\mathcal{S}_{k, \lambda, \beta}^{0, \alpha}(D)$ consisting of one-step skew-product maps.

Definition 3.1 (One-step symbolic cs-blender). Consider a one-step skew-product map $\Phi \in Q$ with $\beta<1$. The maximal invariant set $\Gamma_{\Phi}$ of $\Phi$ in $\Sigma_{k} \times \bar{D}$ is a one-step symbolic cs-blender if there are a non-empty open set $B \subset D$, a fixed point $(\vartheta, p) \in \Sigma_{k} \times D$ of $\Phi$ and a neighborhood $\mathcal{V}$ of $\Phi$ in $Q$ such that for every $\Psi \in \mathcal{V}$ it holds

$$
W^{u}\left(\left(\vartheta, p_{\Psi}\right) ; \Psi\right) \cap\left(W_{l o c}^{s}(\xi ; \tau) \times U\right) \neq \emptyset,
$$

for every $\xi \in \Sigma_{k}$ and every non-empty open subset $U$ in $B$. Here $\left(\vartheta, p_{\Psi}\right)$ is the continuation of the fixed point $(\vartheta, p)$ for $\Psi$.

Observe that Definition 1.5 implies Definition 3.1. Let $(\vartheta, p)$ be a fixed point of $\Phi \in Q$. Note that for $(\xi, x) \in \Sigma_{k} \times B$ and for any $\delta>0$, the set $W_{l o c}^{s}(\xi ; \tau) \times\{x\}$ is a $\delta$-almost horizontal disk. Then by Theorem 2.3 and Definition 1.5, the closure of $W^{u}\left(\left(\vartheta, p_{\Psi}\right) ; \Psi\right)$ meets $W_{l o c}^{s}(\xi ; \tau) \times\{x\}$ for every perturbation $\Psi$ of $\Phi$ in $Q$. Hence the set $W^{u}\left(\left(\vartheta, p_{\Psi}\right) ; \Psi\right)$ meets $W_{l o c}^{s}(\xi ; \tau) \times U$, for every open set $U$ with $x \in U$.

In this section, we prove the existence of symbolic blenders in the one-step setting. We begin studying the relation between one-step skew-products and their associated IFS. Given maps $\phi_{1}, \ldots, \phi_{k}: \bar{D} \rightarrow D$, we denote by $\operatorname{IFS}\left(\phi_{1}, \ldots, \phi_{k}\right)$, the set of all compositions of the maps $\phi_{1}, \ldots, \phi_{k}$ and will refer to this as the associated iterated function system (or IFS) of the one-step map $\Phi=\tau \ltimes\left(\phi_{1}, \ldots, \phi_{k}\right)$. The IFS $(\Phi)=\operatorname{IFS}\left(\phi_{1}, \ldots, \phi_{k}\right)$ has the covering property if there is an open set $B \subset D$ such that

$$
\bar{B} \subset \phi_{1}(B) \cup \cdots \cup \phi_{k}(B) .
$$

To construct symbolic one-step blenders we use the covering property and the Hutchinson attractor of the associated IFS.

3.1. One-step skew-products and IFS's. Consider $\Phi=\tau \ltimes\left(\phi_{1}, \ldots, \phi_{k}\right)$ and let IFS( $\left.\Phi\right)$ be the associated iterated function system. The orbit of a point $x \in M$ for IFS( $\Phi)$ is the set

$$
\operatorname{Orb}_{\Phi}(x) \stackrel{\text { def }}{=}\left\{\phi(x): \phi \in \operatorname{IFS}\left(\phi_{1}, \ldots, \phi_{k}\right)\right\} .
$$

Next proposition shows that if $(\vartheta, p)$ is a fixed point of $\Phi$ then $\operatorname{Orb}_{\Phi}(p)$ is the projection onto the fiber space of the strong unstable set of $(\vartheta, p)$. This result was proved in [NP12, Proposition 2.16]. A consequence of this proposition is that the density property (3.1) of the strong unstable set in Definition 3.1 of one-step symbolic blenders is reduced to the density of the orbit of the "fixed point" $p$ for the associated iteration function system.

Proposition 3.2. Consider $\Phi=\tau \ltimes\left(\phi_{1}, \ldots, \phi_{k}\right)$ and let $(\vartheta, p)$ be a fixed point of $\Phi$. Then $\left.\mathscr{P}\left(W^{u u}(\vartheta, p) ; \Phi\right)\right)=\operatorname{Orb}_{\Phi}(p)$. 
A neighborhood $\mathcal{V}$ of a one-step map $\Phi$ in $Q$ is a neighborhood in the topology of $\mathcal{S}$ intersected with $Q$. Since the topology of $\mathcal{S}$ is induced by the distance in (1.3) and noting that for every $\Psi \in Q$ its Hölder constant is $C_{\Psi}=0$, it follows that $\Psi=\tau \ltimes\left(\psi_{1}, \ldots, \psi_{k}\right)$ and $\Phi=\tau \ltimes\left(\phi_{1}, \ldots, \phi_{k}\right)$ are $\delta$-close if

$$
d_{Q}(\Psi, \Phi)=\max _{i=1, \ldots, k} d_{C^{0}}\left(\left.\psi_{i}\right|_{D},\left.\phi_{i}\right|_{D}\right)<\delta .
$$

A periodic point $(\vartheta, p)$ of a skew-product map $\Phi=\tau \ltimes \phi_{\xi}$ is fiber-hyperbolic for $\Phi$ if $p$ is a hyperbolic fixed point of $\phi_{\vartheta}^{n}$, where $n$ is the period of $(\vartheta, p)$. We analogously define fiber-attractors and fiber-repellors.

Proposition 3.3. Consider $\Phi \in Q$, a non-empty open set $B \subset D$, and a fiber-hyperbolic fixed point $(\vartheta, p) \in \Sigma_{k} \times D$ of $\Phi$. The following properties are equivalent:

i) there is a neighborhood $\mathcal{V}$ of $\Phi$ in $Q$ such that for every $\Psi \in \mathcal{V}$,

$$
W^{u u}\left(\left(\vartheta, p_{\Psi}\right) ; \Psi\right) \cap\left(W_{l o c}^{s}(\xi ; \tau) \times U\right) \neq \emptyset,
$$

for every $\xi \in \Sigma_{k}$ and non-empty open set $U \subset B$. Here $\left(\vartheta, p_{\Psi}\right)$ is the continuation of $(\vartheta, p)$ for $\Psi$.

ii) $B \subset \overline{\operatorname{Orb}_{\Psi}\left(p_{\Psi}\right)}$ for every $\Psi \in Q$ close to $\Phi$.

Proof. From Proposition 3.2, for a fixed point $\left(\vartheta, p_{\Psi}\right)$ of $\Psi$, we have that $\mathscr{P}\left(W^{u u}\left(\left(\vartheta, p_{\Psi}\right) ; \Psi\right)\right)=$ $\mathrm{Orb}_{\Psi}\left(p_{\Psi}\right)$. Therefore, Item (i) implies Item (ii).

For the converse, take the fixed point $\left(\vartheta, p_{\Psi}\right)$ of $\Psi=\tau \ltimes\left(\psi_{1}, \ldots, \psi_{k}\right)$ close to $\Phi=\tau \ltimes$ $\left(\phi_{1}, \ldots, \phi_{k}\right)$ and fix $U \subset B$ and $\xi \in \Sigma_{k}$. By Item (ii), there is $\psi_{i_{n}} \circ \cdots \circ \psi_{i_{1}} \in \operatorname{IFS}(\Psi)$ such that the point $x=\psi_{i_{n}} \circ \ldots \circ \psi_{i_{1}}\left(p_{\Psi}\right) \in U$. Take $\zeta=\left(\ldots \vartheta_{-1} \vartheta_{0}, i_{1}, \ldots, i_{n} ; \xi_{0}, \xi_{1}, \ldots\right)$, and note that $(\zeta, x) \in W_{l o c}^{s}(\xi ; \tau) \times U$. It is enough to see that $(\zeta, x) \in W^{u u}\left(\left(\vartheta, p_{\Psi}\right) ; \Phi\right)$. Since $\left(\vartheta, p_{\Psi}\right)$ is a fixed point of $\Psi$, by the choice of $x$ we have that

$$
\Psi^{-n-1}(\zeta, x)=\left(\left(\ldots, \vartheta_{-1} ; \vartheta_{0}, i_{1}, \ldots, i_{n}, \xi_{0}, \xi_{1}, \ldots\right), p_{\Psi}\right) \in W_{l o c}^{u}(\vartheta ; \tau) \times\left\{p_{\Psi}\right\} .
$$

Since $W_{l o c}^{u}(\vartheta ; \tau) \times\left\{p_{\Psi}\right\}=W_{l o c}^{u u}\left(\left(\vartheta, p_{\Psi}\right) ; \Psi\right)$ then

$$
(\zeta, x) \in \Psi^{n+1}\left(W_{l o c}^{u u}\left(\left(\vartheta, p_{\Psi}\right) ; \Psi\right)\right) \subset W^{u u}\left(\left(\vartheta, p_{\Psi}\right) ; \Psi\right) .
$$

Hence

$$
(\zeta, x) \in W^{u u}\left(\left(\vartheta, p_{\Psi}\right) ; \Psi\right) \cap\left(W_{l o c}^{s}(\xi ; \tau) \times U\right),
$$

completing the proof of the proposition.

Remark 3.4. If $(\vartheta, p)$ in Proposition 3.3 is a fiber-attracting fixed point of $\Phi=\tau \ltimes\left(\phi_{1}, \ldots, \phi_{k}\right)$ with $B$ contained in the attracting region of $p$ for IFS $(\Phi)$, then Item (ii) is equivalent to

$$
B \subset \overline{\operatorname{Orb}_{\Psi}(x)}, \quad \text { for every } x \in B \text { and every } \Psi \in Q \text { close to } \Phi \text {. }
$$


To see why this remark is true first note that Equation (3.2) immediately implies Item (ii) (just take $x=p$ ). For the converse take a perturbation $\Psi=\tau \ltimes\left(\psi_{1}, \ldots, \psi_{k}\right)$ of $\Phi$ in $Q$, a nonempty open set $U$ in $B$, and $x \in B$. By hypotheses, there is $\psi \in \operatorname{IFS}(\Psi)$ such that $\psi\left(p_{\Psi}\right) \in U$. As $U$ is open there is a neighborhood $V$ of $p_{\Psi}$ such that $\psi(V) \subset U$. If $\Psi$ is close enough to $\Phi$ then $B$ is also in the attracting region of $p_{\Psi}$ for $\psi_{\vartheta}=\psi_{i}$ where $\vartheta_{0}=i$. Thus there is $n \in \mathbb{N}$ such that $\psi_{i}^{n}(x) \in V$ and hence $\psi \circ \psi_{i}^{n}(x) \in U$, proving (3.2).

Motivated by (3.2), we give the following definition:

Definition 3.5 (Blending regions). Let $\Phi=\tau \ltimes\left(\phi_{1}, \ldots, \phi_{k}\right) \in Q$. A non-empty open set $B \subset M$ is called a blending region for $\Phi$ (or for the $\operatorname{IFS}(\Phi))$ if for every $\Psi=\tau \ltimes\left(\psi_{1}, \ldots, \psi_{k}\right)$ close to $\Phi$

$$
B \subset \overline{\operatorname{Orb}_{\Psi}(x)} \text { for all } x \in B \text {. }
$$

Proposition 3.6. Let $\Phi=\tau \ltimes\left(\phi_{1}, \ldots, \phi_{k}\right) \in Q$ and consider a blending region $B \subset D$ of $\Phi$. Suppose that there are a hyperbolic fixed point $p \in D$ of some $\phi_{i}$ and a map $\phi \in \operatorname{IFS}(\Phi)$ with $\phi(p) \in B$. Then the maximal invariant set of $\Phi$ in $\Sigma_{k} \times \bar{D}$ is a one-step symbolic blender.

Proof. By Proposition 3.3, it is enough to see that $B \subset \overline{\mathrm{Orb}_{\Psi}\left(p_{\Psi}\right)}$, for every $\Psi=\tau \ltimes\left(\psi_{1}, \ldots, \psi_{k}\right)$ close to $\Phi$, where $p_{\Psi}$ the continuation of $p$ for $\Psi$. By hypothesis, there are $i_{n}, \ldots, i_{1}$ such that $\phi_{i_{n}} \circ \cdots \circ \phi_{i_{1}}(p) \in B$. Since $B$ is an open set, if $\Psi=\tau \ltimes\left(\psi_{1}, \ldots, \psi_{k}\right)$ is close enough to $\Phi$ then $\psi_{i_{n}} \circ \cdots \circ \psi_{i_{1}}\left(p_{\Psi}\right) \in B$. As $B$ is a blending region for $\operatorname{IFS}(\Phi)$ it follows that

$$
B \subset \overline{\operatorname{Orb}_{\Psi}\left(\psi_{i_{n}} \circ \cdots \circ \psi_{i_{1}}\left(p_{\Psi}\right)\right)} \subset \overline{\operatorname{Orb}_{\Psi}\left(p_{\Psi}\right)} .
$$

This concludes the proof of the proposition.

3.2. Blending regions for contracting IFS: Hutchinson attractor. Given an one-step map $\Phi=\tau \ltimes\left(\phi_{1}, \ldots, \phi_{k}\right)$, we define $\operatorname{Per}(\operatorname{IFS}(\Phi))$ as the projection of $\mathscr{P}(\operatorname{Per}(\Phi))$ in the fiber space, that is the set of fixed points of the maps in IFS $(\Phi)$. Associated with $\Phi$ or with $\operatorname{IFS}(\Phi)$, the Hutchinson's operator is defined by

$$
\mathcal{G}_{\Phi}: \mathcal{K}(\bar{D}) \rightarrow \mathcal{K}(\bar{D}), \quad \mathcal{G}_{\Phi}(A) \stackrel{\text { def }}{=} \phi_{1}(A) \cup \cdots \cup \phi_{k}(A),
$$

where $\mathcal{K}(\bar{D})$ denotes the set of compact subsets of $\bar{D}$ and $A \in \mathcal{K}(\bar{D})$. If the maps $\phi_{i}$ are contractions, then the map $\mathcal{G}_{\Phi}$ is also a contraction. This fact leads to the following result:

Proposition 3.7 ([Wil71, Hut81]). Let $\Phi \in Q$ with $\beta<1$. Then there exists a unique compact set $K_{\mathcal{G}_{\Phi}} \in \mathcal{K}(\bar{D})$ such that

$$
K_{\mathcal{G}_{\Phi}}=\mathcal{G}_{\Phi}\left(K_{\mathcal{G}_{\Phi}}\right)=\overline{\operatorname{Per}(\operatorname{IFS}(\Phi)) \cap D}=K_{\Phi}
$$

Moreover, the set $K_{\mathcal{G}_{\Phi}}$ depends continuously (in the set $Q$ ) on the map $\Phi$ and is the global attractor of $\mathcal{G}_{\Phi}$, that is, for every $A \in \mathcal{K}(\bar{D})$ it holds

$$
\lim _{m \rightarrow \infty} d_{H}\left(\mathcal{G}_{\Phi}^{m}(A), K_{\mathcal{G}_{\Phi}}\right)=0 .
$$

We call the compact set $K_{\mathcal{G}_{\Phi}}$ (in what follows denoted by $K_{\Phi}$ ) the Hutchinson attractor of the contracting one-step map $\Phi$ or of its associated IFS $(\Phi)$. 
Let us recall that given $x \in D$, its orbit for $\operatorname{IFS}(\Phi)$ is defined by

$$
\operatorname{Orb}_{\Phi}(x)=\{\phi(x): \phi \in \operatorname{IFS}(\Phi)\}=\left\{\mathcal{G}_{\Phi}^{n}(x): n \geq 0\right\} .
$$

By Proposition 3.7, $\mathcal{G}_{\Phi}^{m}(x) \stackrel{m \rightarrow \infty}{\longrightarrow} K_{\Phi}$ for all $x \in D$ and thus $K_{\Phi} \subset \overline{\mathrm{Orb}_{\Phi}(x)}$. We now have the following straightforward consequences of Proposition 3.7:

Corollary 3.8. Consider $\Phi=\tau \ltimes\left(\phi_{1}, \ldots, \phi_{k}\right) \in Q$ with $\beta<1$ and let $K_{\Phi}$ be the Hutchinson attractor.

i) For every $A \in \mathcal{K}(\bar{D})$ with $A \subset \mathcal{G}_{\Phi}(A)$ it holds that

$$
A \subset K_{\Phi} \subset \overline{\operatorname{Orb}_{\Phi}(x)} \text { for all } x \in \bar{D} \text {. }
$$

ii) For every $p \in K_{\Phi}$ there is a sequence $\left(\sigma_{n}\right)_{n \in \mathbb{N}} \in\{1, \ldots, k\}^{\mathbb{N}}$ such that

$$
\phi_{\sigma_{n}}^{-1} \circ \cdots \circ \phi_{\sigma_{1}}^{-1}(p) \in K_{\Phi} \quad \text { for all } n \in \mathbb{N} \text {. }
$$

iii) For each open set $V$ such that $V \cap K_{\Phi} \neq \emptyset$ there exist $n \in \mathbb{N}$ and $\left(i_{1}, \ldots, i_{n}\right) \in\{1, \ldots, k\}^{n}$ such that $\phi_{i_{n}} \circ \cdots \circ \phi_{i_{1}}\left(K_{\Phi}\right) \subset V$.

These results and Proposition 3.6 imply that the covering property generates one-step symbolic blenders (Definition 3.1):

Corollary 3.9. Consider $\Phi \in Q$ with $\beta<1$. Let $B \subset \bar{D}$ be a non-empty bounded open set satisfying the covering property for $\operatorname{IFS}(\Phi)$. Then for every $\Psi \in Q$ close enough to $\Phi$ one has that $\bar{B} \subset K_{\Psi} \subset \overline{\mathrm{Orb}_{\Psi}(x)}$, for all $x \in \bar{D}$, where $K_{\Psi}$ is the Hutchinson attractor of $\Psi$. Consequently, the maximal invariant set of $\Phi$ is a one-step symbolic blender.

\section{Symbolic blenders in the HöLder Setting}

In this this section we will prove the following result:

Theorem 4.1. Consider $\Phi=\tau \ltimes\left(\phi_{1}, \ldots, \phi_{k}\right) \in \mathcal{S}_{k, \lambda, \beta}^{0, \alpha}(D)$ with $\alpha>0$ and $v^{\alpha}<\lambda<1$ and let $B \subset D$ be an open set. Then, $B$ satisfies the covering property for IFS $(\Phi)$ if and only if there are $\delta>0$ and $a$ neighborhood $\mathcal{V}$ of $\Phi$ in $\mathcal{S}_{k, \lambda, \beta}^{0, \alpha}(D)$ such that for every $\Psi \in \mathcal{V}$ it holds

$$
\Gamma_{\Psi}^{+}(B) \cap H^{s} \neq \emptyset \quad \text { for every } \delta \text {-horizontal disk } H^{s} \text { in } \Sigma_{k} \times B,
$$

where $\Gamma_{\Psi}^{+}(B)$ is the forward maximal invariant set of $\Psi$ in $\Sigma_{k} \times B$.

The above theorem proves Theorem C. Indeed, if $\Phi=\tau \ltimes\left(\phi_{1}, \ldots, \phi_{k}\right) \in \mathcal{S}_{k, \lambda, \beta}^{0, \alpha}(D)$, with $\beta<1$, then $\phi_{i}(\bar{D}) \subset D$ for all $i=1, \ldots, k$. Hence, if $\Psi=\tau \ltimes \psi_{\xi}$ is close enough to $\Phi$ then $\psi_{\xi}(\bar{D}) \subset D$ for all $\xi \in \Sigma_{k}$ and thus

$$
\Gamma_{\Psi}^{+}(B) \stackrel{\text { def }}{=} \bigcap_{n \geq 0} \Psi^{n}\left(\Sigma_{k} \times B\right) \subset \bigcap_{n \in \mathbb{Z}} \Psi^{n}\left(\Sigma_{k} \times \bar{D}\right) \stackrel{\text { def }}{=} \Gamma \Psi .
$$

Since by Theorem 2.3 one has that $W_{l o c}^{u u}\left(\Gamma_{\Psi} ; \Psi\right)=\Gamma_{\Psi}$, Theorem 4.1 and Equation 4.2 imply the existence of symbolic blender (Theorem C).

In order to proof Theorem 4.1, firstly we introduce some notation and preliminary results. 
4.1. Main lemma: Given a finite word $\bar{\omega}=\omega_{-m} \ldots \omega_{-1} \omega_{0} \omega_{1} \ldots \omega_{n}$, where $m, n \geq 0$ and $\omega_{i} \in\{1, \ldots, k\}$, we define the bi-lateral cylinder by

$$
\mathcal{C}_{\bar{\omega}} \stackrel{\text { def }}{=}\left\{\xi \in \Sigma_{k}: \xi_{j}=\omega_{j},-m \leq j \leq n\right\} .
$$

Given $\zeta \in \Sigma_{k}$ and a finite word $\bar{\omega}:=\bar{\omega}_{-n}=\omega_{-n} \ldots \omega_{-1}$, where $n \geq 1$ and $\omega_{i} \in\{1, \ldots, k\}$, we define the relative cylinder by

$$
C_{\bar{\omega}}(\zeta) \stackrel{\text { def }}{=}\left\{\xi \in W_{l o c}^{s}(\zeta ; \tau): \xi_{-i}=\omega_{-i} \text {, for } i=1, \ldots, n\right\} .
$$

Recall that $\mathcal{S}=\mathcal{S}_{k, \lambda, \beta}^{0, \alpha}(D)$ is the set of symbolic skew-product maps in Definition 1.2. In what follows $v^{\alpha}<\lambda<1, \alpha>0$ and there is no restriction on $\beta$. In the next lemma we estimate the distance between the backward orbits of a point $x$ when iterated by different maps $\psi_{\xi}^{-1}$.

Lemma 4.2. Consider $\Psi=\tau \ltimes \psi_{\xi} \in \mathcal{S}$ with $\alpha>0, v^{\alpha}<\lambda<1$ a word $\bar{\omega}=\omega_{-n} \ldots \omega_{0} \ldots \omega_{n}$, and $a$ point $x \in \bar{D}$ such that for every $\zeta \in C_{\bar{\omega}}$ one has that $\psi_{\tau^{-1}(\zeta)}^{-j}(x) \in \bar{D}$ for every $1 \leq j \leq n$. Then

$$
\left\|\psi_{\tau^{-1}(\xi)}^{-i}(x)-\psi_{\tau^{-1}(\zeta)}^{-i}(x)\right\|<C_{\Psi} v^{-\alpha i} \sum_{j=0}^{i-1}\left(\lambda^{-1} v^{\alpha}\right)^{j} d_{\Sigma_{k}}(\xi, \zeta)^{\alpha}
$$

for all $1 \leq i \leq n$ and all $\xi, \zeta \in \mathcal{C}_{\bar{\omega}}$.

Proof. The proof is by induction. For $i=1$, the Hölder inequality (1.2) and $\xi, \zeta \in C_{\bar{\omega}}$ imply

$$
\left\|\psi_{\tau^{-1}(\xi)}^{-1}(x)-\psi_{\tau^{-1}(\zeta)}^{-1}(x)\right\| \leq C_{\Psi} d_{\Sigma_{k}}\left(\tau^{-1}(\xi), \tau^{-1}(\zeta)\right)^{\alpha} \leq v^{-\alpha} d_{\Sigma_{k}}(\xi, \zeta)^{\alpha} .
$$

We argue inductively. Suppose that the lemma holds for $i-1, i<n$ :

$$
\left\|\psi_{\tau^{-1}(\xi)}^{-(i-1)}(x)-\psi_{\tau^{-1}(\zeta)}^{-(i-1)}(x)\right\|<C_{\Psi} v^{-\alpha(i-1)} \sum_{j=0}^{i-2}\left(\lambda^{-1} v^{\alpha}\right)^{j} d_{\Sigma_{k}}(\xi, \zeta)^{\alpha},
$$

for every $\xi, \zeta \in C_{\bar{\omega}}$. We will see that the estimate also holds for $i$. By the triangle inequality,

$$
\begin{gathered}
\left\|\psi_{\tau^{-1}(\xi)}^{-i}(x)-\psi_{\tau^{-1}(\zeta)}^{-i}(x)\right\| \leq\left\|\psi_{\tau^{-1}(\xi)}^{-i}(x)-\psi_{\tau^{-i}(\xi)}^{-1} \circ \psi_{\tau^{-1}(\zeta)}^{-(i-1)}(x)\right\|+ \\
+\left\|\psi_{\tau^{-i}(\xi)}^{-1} \circ \psi_{\tau^{-1}(\zeta)}^{-(i-1)}(x)-\psi_{\tau^{-1}(\zeta)}^{-i}(x)\right\| .
\end{gathered}
$$

Since the inverse of these functions expand at most $1 / \lambda$, the above equation is less than or equal to

$$
\frac{1}{\lambda}\left\|\psi_{\tau^{-1}(\xi)}^{-(i-1)}(x)-\psi_{\tau^{-1}(\zeta)}^{-(i-1)}(x)\right\|+\left\|\psi_{\tau^{-i}(\xi)}^{-1}(y)-\psi_{\tau^{-i}(\zeta)}^{-1}(y)\right\|,
$$

where $y=\psi_{\tau^{-1}(\zeta)}^{-(i-1)}(x) \in \bar{D}$. By the induction hypothesis (4.4),

$$
\frac{1}{\lambda}\left\|\psi_{\tau^{-1}(\xi)}^{-(i-1)}(x)-\psi_{\tau^{-1}(\zeta)}^{-(i-1)}(x)\right\| \leq C_{\Psi} \lambda^{-1} v^{-\alpha(i-1)} \sum_{j=0}^{i-2}\left(\lambda^{-1} v^{\alpha}\right)^{j} d_{\Sigma_{k}}(\xi, \zeta)^{\alpha} .
$$

As $y \in \bar{D}$, applying the Hölder inequality (1.2) and since $\xi, \zeta \in \mathcal{C}_{\bar{\omega}}$ we get

$$
\left\|\psi_{\tau^{-i}(\xi)}^{-1}(y)-\psi_{\tau^{-i}(\zeta)}^{-1}(y)\right\| \leq C_{\Psi} d \Sigma_{\Sigma_{k}}\left(\tau^{-i}(\xi), \tau^{-i}(\zeta)\right)^{\alpha} \leq C_{\Psi} v^{-\alpha i} d \Sigma_{k}(\xi, \zeta)^{\alpha} .
$$


Putting together the previous inequalities we obtain

$$
\begin{gathered}
C_{\Psi} \lambda^{-1} v^{-\alpha(i-1)} \sum_{j=0}^{i-2}\left(\lambda^{-1} v^{\alpha}\right)^{j} d_{\Sigma_{k}}(\xi, \zeta)^{\alpha}+C_{\Psi} v^{-\alpha i} d_{\Sigma_{k}}(\xi, \zeta)^{\alpha} \\
=C_{\Psi} v^{-\alpha i} \sum_{j=0}^{i-1}\left(\lambda^{-1} v^{\alpha}\right)^{j} d_{\Sigma_{k}}(\xi, \zeta)^{\alpha},
\end{gathered}
$$

ending the proof of the lemma.

4.2. Proof of Theorem 4.1. Consider $\Phi=\tau \ltimes\left(\phi_{1}, \ldots, \phi_{k}\right) \in \mathcal{S}$ with $\alpha>0, v^{\alpha}<\lambda<1$ and an open set $B \subset D$. Recall that we need to prove the following:

$B$ satisfies the covering property for IFS $(\Phi) \Longleftrightarrow$ there are $\delta>0$ and a neighborhood $\mathcal{V}$ of $\Phi$ in $\mathcal{S}$ such that $\Gamma_{\Psi}^{+}(B) \cap H^{s} \neq \emptyset$ for every $\Psi \in \mathcal{V}$ and every $\delta$-horizontal disk $H^{s}$ in $\Sigma_{k} \times B$.

$\Longleftarrow$ We will see that if the covering property is not satisfied then intersection (4.1) is also not satisfied. If $B$ does not satisfy the covering property then there is $x \in \bar{B}$ such that $x \notin \phi_{i}(B)$ for all $i=1, \ldots, k$. We can assume that $x \in B$, otherwise we can take an arbitrarily small perturbation $\Psi=\tau \ltimes\left(\psi_{1}, \ldots, \psi_{k}\right)$ of $\Phi$ such that the covering property in $B$ for IFS( $\left.\Psi\right)$ is not satisfied for a point in $B$. The condition $x \notin \phi_{i}(B)$ for all $i=1, \ldots, k$ implies that $\Phi^{-1}(\xi, x) \notin \Sigma_{k} \times B$ for all $\xi \in \Sigma_{k}$ and hence

$$
(\xi, x) \notin \bigcap_{n \geq 0} \Phi^{n}\left(\Sigma_{k} \times B\right)=\Gamma_{\Phi}^{+}(B) \quad \text { for all } \xi \in \Sigma_{k} .
$$

Therefore $\Gamma_{\Phi}^{+}(B)$ does not meet the horizontal disk $H^{s}=W_{l o c}^{s}(\xi ; \tau) \times\{x\}$, and thus the intersection property (4.1) is not verified.

$\Longrightarrow$ We split the proof of the fact that the covering property implies the intersection condition into two steps.

Choice of the neighborhood $\mathcal{V}$ of $\Phi$. Recall that given an open covering $C$ of a compact set $X$ of a metric space, there is a constant $L>0$, called Lebesgue number of $C$, such that every subset of $X$ with diameter less than $L$ is contained in some member of $C$.

Let $2 L>0$ be a Lebesgue number of the open covering $\left\{\phi_{1}(B), \ldots, \phi_{k}(B)\right\}$ of $\bar{B}$. Note that there are $C^{0}$-neighborhoods $\mathcal{U}_{i}$ of $\phi_{i}$ such that the family

$$
B_{i}=\operatorname{int}\left(\bigcap_{\psi \in \mathcal{U}_{i}} \psi(B)\right), \quad i=1, \ldots, k,
$$

is an open covering of $\bar{B}$. By shrinking the size of the sets $\mathcal{U}_{i}$ we can assume that $L$ is a Lebesgue number of this covering. We can also assume that any $\psi \in \mathcal{U}_{i}$ is a $C^{0}-(\lambda, \beta)$ Lipschitz map on $\bar{D}$ for all $i=1, \ldots, k$.

We take a neighborhood $\mathcal{V}$ of $\Phi$ in $\mathcal{S}$ such that if $\Psi=\tau \ltimes \psi_{\xi} \in \mathcal{V}$ then $\psi_{\xi} \in \mathcal{U}_{i}$ with $i=\xi_{0}$. By (4.5),

$$
\psi_{\tau^{-1}(\xi)}^{-1}\left(\overline{B_{i}}\right) \subset B \quad \text { for all } \xi \in \Sigma_{k} \text { with } \xi_{-1}=i \text {. }
$$


Since $\Phi$ is a one-step map then $\phi_{\xi}=\phi_{\zeta}$ if $\xi_{0}=\zeta_{0}$, and hence we can take the Hölder constant $C_{\Phi}=0$. The definition of the distance in (1.3) implies $C_{\Psi}$ is close to $C_{\Phi}=0$. By hypothesis $v^{\alpha}<\lambda$, and so shrinking the neighborhood $\mathcal{V}$ we can assume that for every $\Psi=\tau \ltimes \psi_{\xi} \in \mathcal{V}$,

$$
C_{\Psi} \sum_{i=0}^{\infty}\left(\lambda^{-1} v^{\alpha}\right)^{i}<L / 2 .
$$

This completes the choice of the neighborhood $\mathcal{V}$ of $\Phi$.

Existence of a point in $\Gamma_{\Psi}^{+}(B) \cap H^{s}$. Let us consider $\mathcal{V}$ to be the above neighborhood of $\Phi$. The main step is the following proposition.

Proposition 4.3. Consider $0<\delta<\lambda L / 2$ and let $H^{s}$ be a $\delta$-horizontal disk in $\Sigma_{k} \times B$ associated with some $(\zeta, z) \in \Sigma_{k} \times B$ and $\alpha$-Hlder constant $C \geq 0$. Then for every $\Psi=\tau \ltimes \psi_{\xi} \in \mathcal{V}$ there are an infinite word $\bar{\omega}=\ldots \omega_{-n} \ldots \omega_{-1}$ with $\omega_{-n} \in\{1, \ldots, k\}$ and a sequence of nested compacts subsets $\left\{V_{n}\right\}$ of $M$ such that for every $n \in \mathbb{N}$ it holds that

$$
\begin{gathered}
V_{n} \subset \mathscr{P}\left(H^{s} \cap\left(C_{\bar{\omega}_{-n}}(\zeta) \times B\right)\right), \\
\psi_{\tau^{-1}(\xi)}^{-n}\left(V_{n}\right) \subset B \text { and } \operatorname{diam}\left(\psi_{\tau^{-1}(\xi)}^{-n}\left(V_{n}\right)\right) \leq C\left(\lambda^{-1} v^{\alpha}\right)^{n}
\end{gathered}
$$

for all $\xi \in C_{\bar{\omega}_{-n}}(\zeta)$ where $\bar{\omega}_{-n}=\omega_{-n} \ldots \omega_{-1}$.

Let us see how the implication $(\Longrightarrow)$ follows from this proposition. Let

$$
\{x\}=\bigcap_{n \in \mathbb{N}} V_{n} \subset B \quad \text { and } \quad\{\xi\}=\bigcap_{n \in \mathbb{N}} C_{\bar{\omega}_{-n}}(\zeta) \subset W_{l o c}^{s}(\zeta ; \tau) \text {. }
$$

Observe that $(\xi, x) \in H^{s}$ and $\psi_{\tau^{-1}(\xi)}^{-n}(x) \in B$ for all $n \in \mathbb{N}$. Thus $\Psi^{-n}(\xi, x) \in \Sigma_{k} \times B$ for all $n \in \mathbb{N}$ and hence $(\xi, x) \in \Gamma_{\Psi}^{+}(B)$. Therefore $\Gamma_{\Psi}^{+}(B) \cap H^{s} \neq \emptyset$.

To complete the proof of Theorem 4.1 it remains to prove the proposition.

Proof of Proposition 4.3. Fix $\Psi=\tau \ltimes \psi_{\xi} \in \mathcal{V}$ and consider the $(\alpha, C)$-Hlder map $h: W_{l o c}^{s}(\zeta ; \tau) \rightarrow B$ associated with the $\delta$-horizontal disk $H^{s} \subset \Sigma_{k} \times B$ (see Definition 1.4). The construction of the nested sequence of sets $\left\{V_{n}\right\}$ and the infinite word $\bar{\omega}=\ldots \omega_{-m} \ldots \omega_{-1}$ is done inductively. Let

$$
V \stackrel{\text { def }}{=} \mathscr{P}\left(H^{s}\right) \subset B .
$$

Note that $\operatorname{diam}(V) \leq 2 \delta<L$. By the definition of the Lebesgue number, we have that $V \subset B_{i_{1}}$ for some $i_{1} \in\{1, \ldots, k\}$. Recall the definition of the relative cylinder in (4.3) associated with $\zeta \in \Sigma_{k}$ and the word $\bar{\omega}_{-1}=i_{1}$ and consider the set

$$
V_{1} \stackrel{\text { def }}{=} \mathscr{P}\left(H^{s} \cap\left(C_{\bar{\omega}_{-1}}(\zeta) \times V\right)\right) \text {. }
$$

By construction $V_{1} \subset V \subset B_{i_{1}}$ and by (4.6),

$$
\psi_{\tau^{-1}(\xi)}^{-1}\left(V_{1}\right) \subset B \quad \text { for all } \xi \in C_{\bar{\omega}_{-1}}(\zeta) .
$$

Claim 4.4. $\operatorname{diam}\left(V_{1}\right) \leq \delta_{1} \stackrel{\text { def }}{=} C v^{\alpha}$. 
Proof. Given $x$ and $y$ in $V_{1}$ there are $\xi$ and $\eta$ in $C_{\bar{\omega}_{-1}}(\zeta)$ such that $x=h(\xi)$ and $y=h(\eta)$. Since $h$ is $(\alpha, C)$-Hölder continuous,

$$
\|x-y\|=\|h(\xi)-h(\eta)\| \leq C d_{\Sigma_{k}}(\xi, \eta)^{\alpha} \leq C v^{\alpha}=\delta_{1},
$$

proving the claim.

By Claim 4.4 and since the fiber-maps $\psi_{\xi}$ are $(\lambda, \beta)$-Lipschitz on $\bar{D}$,

$$
\operatorname{diam}\left(\psi_{\tau^{-1}(\xi)}^{-1}\left(V_{1}\right)\right) \leq \lambda^{-1} \delta_{1} \quad \text { for all } \xi \in \mathcal{C}_{\bar{\omega}_{-1}}(\zeta) .
$$

Recalling that $C v^{\alpha}<\delta$ (see Definition 1.4) we get that

$$
\lambda^{-1} \delta_{1}=\lambda^{-1} C v^{\alpha}<\lambda^{-1} \delta \leq L / 2 \text {. }
$$

Therefore,

$$
\operatorname{diam}\left(\psi_{\tau^{-1}(\xi)}^{-1}\left(V_{1}\right)\right) \leq \lambda^{-1} \delta_{1} \leq L / 2
$$

Arguing inductively, we suppose that we have constructed a finite word $\bar{\omega}_{-n}:=\omega_{-n} \ldots \omega_{-1}$ (the word $\bar{\omega}_{-i}$ is obtained adding the letter $\omega_{-i}$ to the word $\bar{\omega}_{-i+1}$ ) and closed sets $V_{n} \subset V_{n-1} \subset$ $\cdots \subset V_{1}$ with

$$
\operatorname{diam}\left(V_{n}\right) \leq C v^{n \alpha} \stackrel{\text { def }}{=} \delta_{n}
$$

and such that for every $\xi \in C_{\bar{\omega}_{-n}}(\zeta)$,

$$
\psi_{\tau^{-1}(\xi)}^{-n}\left(V_{n}\right) \subset B \text { and } \operatorname{diam}\left(\psi_{\tau^{-1}(\xi)}^{-n}\left(V_{n}\right)\right) \leq \lambda^{-n} \delta_{n} .
$$

We now construct the word $\bar{\omega}_{-(n+1)}$ and the closed set $V_{n+1} \subset V_{n}$ satisfying analogous inclusions and inequalities. By (4.8) we have that

$$
A_{n} \stackrel{\text { def }}{=} \bigcup_{\xi \in \mathcal{C}_{\bar{\omega}-n}(\zeta)} \psi_{\tau^{-1}(\xi)}^{-n}\left(V_{n}\right) \subset B
$$

Claim 4.5. $\operatorname{diam}\left(A_{n}\right)<L$.

Proof. Given $\bar{x}$ and $\bar{y}$ in $A_{n}$ there are $x, y \in V_{n}$ and $\xi, \eta \in C_{\bar{\omega}_{-n}}(\zeta)$ such that $\bar{x}=\psi_{\tau^{-1}(\xi)}^{-n}(x)$ and $\bar{y}=\psi_{\tau^{-1}(\eta)}^{-n}(y)$. Then

$$
\begin{aligned}
\|\bar{x}-\bar{y}\| & =\left\|\psi_{\tau^{-1}(\xi)}^{-n}(x)-\psi_{\tau^{-1}(\eta)}^{-n}(y)\right\| \\
& \leq\left\|\psi_{\tau^{-1}(\xi)}^{-n}(x)-\psi_{\tau^{-1}(\eta)}^{-n}(x)\right\|+\left\|\psi_{\tau^{-1}(\eta)}^{-n}(x)-\psi_{\tau^{-1}(\eta)}^{-n}(y)\right\| \\
& \leq C_{\Psi} v^{-\alpha n} \sum_{j=0}^{n-1}\left(\lambda^{-1} v^{\alpha}\right)^{j} d_{\Sigma_{k}}(\xi, \eta)^{\alpha}+\lambda^{-n} \delta_{n}
\end{aligned}
$$

where the last inequality is follows from Lemma 4.2 and the induction hypothesis (4.8). Since $\xi$ and $\eta$ belong to $C_{\bar{\omega}_{-n}}(\zeta)$ then $d_{\Sigma_{k}}(\xi, \eta)^{\alpha} \leq v^{\alpha n}$. Hence

$$
\|\bar{x}-\bar{y}\| \leq C_{\Psi} \sum_{j=0}^{n-1}\left(\lambda^{-1} v^{\alpha}\right)^{j}+\lambda^{-n} \delta_{n} \leq L / 2+\lambda^{-n} \delta_{n}
$$

where the last inequality follows from (4.7). Note that

$$
\lambda^{-n} \delta_{n}=C\left(\lambda^{-1} v^{\alpha}\right)^{n} \leq C \lambda^{-1} v^{\alpha}<\lambda^{-1} \delta<L / 2 .
$$


Therefore for every pair of points $\bar{x}, \bar{y} \in A_{n}$ we have $\|\bar{x}-\bar{y}\|<L$ and thus $\operatorname{diam}\left(A_{n}\right)<L$, proving the claim.

Since $L$ is a Lebesgue number of the covering $\left\{B_{i}\right\}_{i=1}^{k}$, the claim implies there is $i_{n+1} \in$ $\{1, \ldots, k\}$ such that $A_{n} \subset B_{i_{n+1}}$. We let

$$
\bar{\omega}_{-(n+1)}=i_{n+1} \omega_{-n} \ldots \omega_{-1} \quad \text { and } \quad V_{n+1}=\mathscr{P}\left(H^{s} \cap\left(C_{\bar{\omega}_{-(n+1)}}(\zeta) \times V_{n}\right)\right) \text {. }
$$

Note that by construction $V_{n+1} \subset V_{n}$.

Claim 4.6. $\operatorname{diam}\left(V_{n+1}\right) \leq C v^{(n+1) \alpha} \stackrel{\text { def }}{=} \delta_{n+1}$.

Proof. Given $x, y \in V_{n+1}$ there are $\xi, \eta \in C_{\bar{\omega}_{-(n+1)}}(\zeta)$ such that $x=h(\xi)$ and $y=h(\eta)$. From the $(\alpha, C)$-Hölder continuity of $h$ and since $\xi, \eta \in C_{\bar{\omega}_{-(n+1)}}(\zeta)$ we get $\|x-y\| \leq C d_{\Sigma_{k}}(\xi, \eta)^{\alpha} \leq C v^{(n+1) \alpha}$, concluding the proof.

Using that $V_{n+1} \subset V_{n}, \operatorname{diam}\left(V_{n+1}\right) \leq \delta_{n+1}$, and equations (4.6) and (4.8) we get

$$
\psi_{\tau^{-1}(\xi)}^{-(n+1)}\left(V_{n+1}\right) \subset B \quad \text { and } \operatorname{diam}\left(\psi_{\tau^{-1}(\xi)}^{-(n+1)}\left(V_{n+1}\right)\right) \leq \lambda^{-(n+1)} \delta_{n+1},
$$

for all $\xi \in C_{\bar{\omega}_{-(n+1)}}(\zeta)$. Thus (4.8) holds for $(n+1)$-step and we can continue arguing inductively. This completes the construction of the infinite word $\bar{\omega}$ and the sequence of nested sets $\left\{V_{n}\right\}$ in the proposition, ending the proof.

The proof of Theorem 4.1 is now complete.

4.3. Embedded blender. For applications of blenders we would need to have additional fiber maps, that are not necessarily contracting or forward invariant in the region $D$. Thus, we will embed a symbolic blender for a one-step map $\Phi$ defined on $\Sigma_{k} \times M$ into another one-step map $\hat{\Phi}$ on $\Sigma_{d} \times M$ with $d \geq k$.

Proposition 4.7 (Embedded blender). Let $\hat{\Phi} \in \mathcal{P} \mathcal{H} \mathcal{S}_{d}^{0, \alpha}(M)$ be a partially hyperbolic one-step skew-product map with $d \geq k$. Assume that the restriction

$$
\Phi=\left.\hat{\Phi}\right|_{\Sigma_{k} \times M}=\tau \ltimes\left(\phi_{1}, \ldots, \phi_{k}\right)
$$

has a symbolic cs-blender $\Gamma_{\Phi}^{c s} \subset \Sigma_{k} \times M$ whose superposition domain contains an open set $B \subset M$ satisfying that $\bar{B} \subset \phi_{1}(B) \cup \cdots \cup \phi_{k}(B)$. Then there exists $\delta>0$ such that for every small enough $\mathcal{S}^{0, \alpha}$-perturbation $\hat{\Psi}$ of $\hat{\Phi}$ it holds

$$
W_{\text {loc }}^{u u}\left(\Gamma_{\Psi}^{c s} ; \hat{\Psi}\right) \cap H^{s} \neq \emptyset \quad \text { for every } \delta \text {-horizontal disk } H^{s} \text { in } \Sigma_{d} \times B
$$

where $\Gamma_{\Psi}^{c s}$ is the continuation of $\Gamma_{\Phi}^{c s}$ for $\Psi=\left.\hat{\Psi}\right|_{\Sigma_{k} \times M}$.

Proof. Since $\Gamma_{\Phi}^{c s}$ is a symbolic blender for $\Phi$ whose superposition domain contains the open set $B$ then $\Gamma_{\Phi}^{+}(B) \subset \Gamma_{\Phi}^{c s}$. Recall that $\Gamma_{\Phi}^{+}(B)$ is the forward maximal invariant set in $\Sigma_{k} \times B$ for $\Phi$. We can enlarge the size of $B$ a little, call this new set $\tilde{B}$, assuming that for every small enough $\mathcal{S}^{0, \alpha}$-perturbation $\hat{\Psi}$ of $\hat{\Phi}$, it holds

$$
\Gamma_{\Psi}^{+}(\tilde{B}) \subset \Gamma_{\Psi \prime}^{c s}, \quad \text { where } \Psi=\left.\hat{\Psi}\right|_{\Sigma_{k} \times M} .
$$


Observe that for each $(\xi, x) \in \Sigma_{d} \times M$, since $\hat{\Phi}$ is a one-step map then

$$
W_{l o c}^{u u}((\xi, x) ; \hat{\Phi})=W_{l o c}^{u}(\xi ; \tau) \times\{x\} .
$$

Since the strong unstable set depends continuously on $\hat{\Phi}$, we can assume that for every small enough $\mathcal{S}^{0, \alpha}$-perturbation $\hat{\Psi}$ of $\hat{\Phi}$,

$$
W_{l o c}^{u u}((\xi, x) ; \hat{\Psi}) \subset \Sigma_{d} \times \tilde{B} \quad \text { for all }(\xi, x) \in \Sigma_{d} \times B \text {. }
$$

A slight modification of the proof of Theorem 4.1 shows that there is $\delta>0$ such that for every small enough $\mathcal{S}^{0, \alpha}$-perturbation $\hat{\Psi}$ of $\hat{\Phi}$ we have

$$
\Gamma_{\hat{\Psi}}^{+}\left(\Sigma_{k, d}^{-} \times B\right) \cap H^{s} \neq \emptyset
$$

for every $\delta$-horizontal disk $H^{s}$ in $\Sigma_{d} \times B$. Here

$$
\Gamma_{\hat{\Psi}}^{+}\left(\Sigma_{k, d}^{-} \times B\right)=\bigcap_{n \geq 0} \hat{\Psi}^{n}\left(\Sigma_{k, d}^{-} \times B\right)
$$

and $\Sigma_{k, d}^{-}=\left\{\xi=\left(\xi_{i}\right)_{i \in \mathbb{Z}} \in \Sigma_{d}: \xi_{i} \in\{1, \ldots, k\}\right.$ for $\left.i<0\right\}$. This assertion is showed by repeating the same argument as in the proof of Theorem 4.1, using the global s-domination condition $v^{\alpha}<\lambda$ and the global Hlder continuity of the fiber maps with respect to the base point.

Consider a $\mathcal{S}^{0, \alpha}$-perturbation $\hat{\Psi}=\tau \ltimes \psi_{\xi}$ of $\hat{\Phi}$ satisfying (4.9), (4.10) and (4.11) and let $\Gamma_{\Psi}^{c s} \subset \Sigma_{k} \times M$ be the continuation of $\Gamma_{\Phi}^{c s}$ for $\Psi=\left.\hat{\Psi}\right|_{\Sigma_{k} \times M}$.

Claim 4.8. $\Gamma_{\hat{\Psi}}^{+}\left(\Sigma_{k, d}^{-} \times B\right) \subset W_{l o c}^{u u}\left(\Gamma_{\Psi}^{c s} ; \hat{\Psi}\right)$.

Proof. Fix a point $(\xi, x) \in \Gamma_{\hat{\Psi}}^{+}\left(\Sigma_{k, d}^{-} \times B\right)$ we show that

$$
W_{l o c}^{u u}((\xi, x) ; \hat{\Psi}) \cap\left(\Sigma_{k} \times \tilde{B}\right) \subset \Gamma_{\Psi}^{c S} .
$$

This assertion proves the claim since if $(\zeta, z) \in W_{l o c}^{u u}((\xi, x) ; \hat{\Psi}) \cap\left(\Sigma_{k} \times \tilde{B}\right)$ then $(\xi, x) \in W_{l o c}^{u u}((\zeta, z) ; \hat{\Psi}) \subset$ $W_{l o c}^{u u}\left(\Gamma_{\Psi}^{c s} ; \hat{\Psi}\right)$.

Let $(\zeta, z) \in W_{l o c}^{u u}((\xi, x) ; \hat{\Psi}) \cap\left(\Sigma_{k} \times \tilde{B}\right)$. In order to prove (4.12), observe that from (4.9) it is enough to show that $\hat{\Psi}^{-n}(\zeta, z)=\Psi^{-n}(\zeta, z) \in \Sigma_{k} \times \tilde{B}$ for all $n \geq 0$. From the invariance of the local strong unstable lamination (dual statement of Item (iii) in Proposition 2.1) it follows that

$$
\hat{\Psi}^{-n}(\zeta, z) \in \hat{\Psi}^{-n}\left(W_{l o c}^{u u}((\xi, x) ; \hat{\Psi})\right) \subset W_{l o c}^{u u}\left(\hat{\Psi}^{-n}(\xi, x) ; \hat{\Psi}\right)
$$

for all $n \geq 0$. Since $(\xi, x) \in \Gamma_{\hat{\Psi}}^{+}\left(\Sigma_{k, d}^{-} \times B\right)$ then $\hat{\Psi}^{-n}(\xi, x) \in \Sigma_{d} \times B$ for all $n \geq 0$ and according to $(4.10)$ it follows that

$$
W_{l o c}^{u u}\left(\hat{\Psi}^{-n}(\xi, x) ; \hat{\Psi}\right) \subset \Sigma_{d} \times \tilde{B} \quad \text { for all } n \geq 0 .
$$

Hence, since $\zeta \in \Sigma_{k}$, (4.13) and (4.14) imply that $\hat{\Psi}^{-n}(\zeta, z) \in \Sigma_{k} \times \tilde{B}$ for all $n \geq 0$. Therefore $(\zeta, z) \in \Gamma_{\Psi}^{+}(\tilde{B}) \subset \Gamma_{\Psi}^{c S}$ concluding (4.12) and the proof of the claim.

To conclude the proposition it suffices to observe that the above claim and (4.11) imply that $W_{l o c}^{u u}\left(\Gamma_{\Psi}^{c s} ; \hat{\Psi}\right) \cap H^{s} \neq \emptyset$ for every $\delta$-horizontal disk $H^{s}$ in $\Sigma_{d} \times B$. The proof is now complete. 
Observe that the set $W_{l o c}^{s}(\zeta ; \tau) \times\{z\}$ is an horizontal disk and in the one-step case this set coincides to the local strong stable set of $(\zeta, z)$. For Hölder perturbations of one-step maps, Proposition 2.1 implies that the local strong stable sets are almost horizontal disks. Then, we obtain the following remark:

Remark 4.9. Let $\hat{\Phi} \in \mathcal{P} \mathcal{H} \mathcal{S}_{d}^{0, \alpha}(M)$ be a one-step skew-product map as in the hypothesis of Proposition 4.7. For every small enough $\mathcal{S}^{0, \alpha}$-perturbation $\hat{\Psi}$ of $\hat{\Phi}$ and every $(\xi, x) \in \Sigma_{d} \times B$ there exits $(\zeta, z) \in \Gamma_{\Psi}^{c s}$ such that

$$
W_{l o c}^{u u}((\zeta, z) ; \hat{\Psi}) \cap W_{l o c}^{s s}((\xi, x) ; \hat{\Psi}) \neq \emptyset .
$$

4.4. $\mathrm{cu}$-blenders. Let us now define a symbolic $c u$-blender-horseshoe. Firstly we need to introduce the associated inverse symbolic skew-product for $\Phi=\tau \ltimes \phi_{\xi}$. Given $\Phi=\tau \ltimes \phi_{\xi} \in$ $\mathcal{S}_{k, \lambda, \beta}^{0, \alpha}(D)$, the symbolic skew-product

$$
\Phi^{*}=\tau \ltimes \phi_{\xi}^{*} \in \mathcal{S}_{k, \beta^{-1}, \lambda^{-1}}^{0, \alpha}(D),
$$

where $\phi_{\xi}^{*}: M \rightarrow M$ given by $\phi_{\xi}^{*}(x)=\phi_{\xi^{*}}^{-1}(x)$, is called the associated inverse skew-product for $\Phi$. Here $\xi^{*}=\left(\ldots \xi_{1} ; \xi_{0}, \xi_{-1}, \ldots\right)$ denotes the conjugate sequence of $\xi=\left(\ldots \xi_{-1} ; \xi_{0}, \xi_{1}, \ldots\right)$. Note that since $\tau(\xi)^{*}=\tau^{-1}\left(\xi^{*}\right)$, then iterates of $\Phi^{*}$ correspond to iterates of $\Phi^{-1}$. This observation allows us to define symbolic $c u$-blender-horseshoes for skew-products in $\mathcal{S}_{k, \lambda, \beta}^{0, \alpha}(D)$ with $\lambda>1$ and $\alpha>0$. Namely, symbolic cu-blender-horseshoe for $\Phi$ is defined as a symbolic cs-blenderhorseshoe for $\Phi^{*}$.

One then obtains analogous results to Theorem 4.1 and Proposition 4.7 for the construction of $c u$-blenders using the inverse covering property, $\bar{B} \subset \phi_{1}^{-1}(B) \cup \cdots \cup \phi_{k}^{-1}(B)$.

\section{Robust HETERODIMENSIONAL CYCLES AND MIXING SETS}

Our goal here is to generate robust heterodimensional cycles (Section 5.1) and robust nonhyperbolic mixing sets (Section 5.2) in the presence of symbolic blenders. Let us comment on the strategy used in the proof. The idea is to give conditions on the IFS (covering property) which will imply that the associated one-step skew-product map has a symbolic cycle or is mixing (see Definition 5.1). Then, one studies the robustness of these properties under perturbations in the symbolic setting (see Theorems 5.2 and 5.7). Finally, the smooth realization of the one-step skew-product shows that the corresponding property is robust in the $C^{1}$-topology (Proposition A.2 in appendix).

In this section, $N$ and $M$ denote compact Riemannian manifolds and id: $M \rightarrow M$ the identity map.

5.1. Robust heterodimensional cycles: Proof of Theorem A. We will first describe how to build robust cycles in the symbolic setting and then transfer this to dynamics of diffeomorphisms on manifolds, thus proving Theorem A. 
Definition 5.1 (Symbolic cycles). Let $\hat{\Phi} \in \mathcal{P} \mathcal{H} \mathcal{S}_{d}^{r, \alpha}(M)$ be a symbolic skew-product with a pair of $\hat{\Phi}$-invariant sets $\Gamma_{1}$ and $\Gamma_{2}$. We say that $\hat{\Phi}$ has a symbolic cycle associated with $\Gamma_{1}$ and $\Gamma_{2}$ if their stable/unstable sets meet cyclically, that is, if

$$
W^{s}\left(\Gamma_{1} ; \hat{\Phi}\right) \cap W^{u}\left(\Gamma_{2} ; \hat{\Phi}\right) \neq \emptyset \quad \text { and } \quad W^{u}\left(\Gamma_{1} ; \hat{\Phi}\right) \cap W^{s}\left(\Gamma_{2} ; \hat{\Phi}\right) \neq \emptyset .
$$

Assuming that $\Gamma_{1}$ and $\Gamma_{2}$ have continuations, we say that the symbolic cycle is $\mathcal{S}^{r, \alpha}$-robust if the cyclic intersection of stable/unstable sets holds for every small enough $\mathcal{S}^{r, \alpha}$-perturbation of $\hat{\Phi}$.

The next proposition shows how to construct robust symbolic cycles using blenders that come from the covering property.

Theorem 5.2 (Symbolic cycles from blenders). Let $\phi_{1}, \ldots, \phi_{k}, \phi_{k+1}, \phi_{k+2}$ be $\left(\gamma, \hat{\gamma}^{-1}\right)$-Lipschitz $C^{1}$-diffeomorphisms on $M$ and consider disjoint open sets $D_{c s}, D_{c u} \subset M$ such that

$$
\Phi=\tau \ltimes\left(\phi_{1}, \ldots, \phi_{k}\right) \in \mathcal{S}_{k, \lambda_{c s}, \beta_{c s}}^{1, \alpha}\left(D_{c s}\right) \cap \mathcal{S}_{k, \lambda_{c u}, \beta_{c u}}^{1, \alpha}\left(D_{c u}\right)
$$

where

$$
v^{\alpha}<\gamma \leq \lambda_{c s}<\beta_{c s}<1<\lambda_{c u}<\beta_{c u} \leq \hat{\gamma}^{-1}<v^{-\alpha} .
$$

Assume that there are open subsets $B_{c s} \subset D_{c s}$ and $B_{c u} \subset D_{c u}$ such that the following properties hold:

- Covering property:

$$
\overline{B_{c s}} \subset \bigcup_{i=1}^{k} \phi_{i}\left(B_{c s}\right) \text { and } \overline{B_{c u}} \subset \bigcup_{i=1}^{k} \phi_{i}^{-1}\left(B_{c u}\right)
$$

- Cyclic intersections: there exist $x \in B_{c s}, y \in D_{c u}$ and $m, n>0$ such that

$$
\phi_{k+1}^{n}(x) \in B_{c u} \text { and } \phi_{k+2}^{m}(y) \in D_{c s} .
$$

Then the one-step $\hat{\Phi}=\tau \ltimes\left(\phi_{1}, \ldots, \phi_{k}, \phi_{k+1}, \phi_{k+2}\right) \in \mathcal{P} \mathcal{H} \mathcal{S}_{k+2}^{1, \alpha}(M)$ has a $\mathcal{S}^{1, \alpha}$-robust symbolic cycle associated with symbolic blenders whose superposition domains contain $B_{c s}$ and $B_{c u}$.

Proof. We split the proof of the proposition into two lemmas. Firstly, we need to choose the $\mathcal{S}^{1, \alpha}$-neighborhood $\mathcal{V}$ of $\hat{\Phi}=\tau \ltimes\left(\phi_{1}, \ldots, \phi_{k}, \phi_{k+1}, \phi_{k+2}\right)$ in which we will work. This neighborhood is taken as the intersection of the following three sets:

- $\mathcal{V}_{1}$ is the neighborhood of $\hat{\Phi}$ given by Proposition 4.7 .

- $\mathcal{V}_{2}$ is the neighborhood of $\hat{\Phi}$ such that for every $\hat{\Psi} \in \mathcal{V}_{2}$ and $y \in D_{c u}$ (from the cyclic intersection hypothesis) it holds

$$
\hat{\Psi}^{m}\left(C^{+} \times\{y\}\right) \subset \Sigma_{k+2} \times D_{c s}
$$

where $C^{+}$is the set of the bi-sequence $\xi=\left(\xi_{i}\right)_{i \in \mathbb{Z}} \in \Sigma_{k+2}$ such that $\xi_{i}=k+2$ for $0 \leq i \leq m$ and $\xi_{i} \in\{1, \ldots, k\}$ otherwise.

- $\mathcal{V}_{3}$ is the neighborhood of $\hat{\Phi}$ taken so that the strong stable sets are $\delta$-almost horizontal disks, where $\delta>0$ is defined in the following manner. Suppose $z \in M$ with $\| z-$ $\phi_{k+1}^{n}(x) \|<\delta$, then for every $\hat{\Psi} \in \mathcal{V}_{3}$ it holds

$$
\hat{\Psi}^{-n}\left(C^{-} \times\{z\}\right) \subset \Sigma_{k+2} \times B_{c s}
$$


where $C^{-}$is the set of the bi-sequences $\xi=\left(\xi_{i}\right)_{i \in \mathbb{Z}} \in \Sigma_{k+2}$ such that $\xi_{i}=k+2$ for $-n \leq i<0$ and $\xi_{i} \in\{1, \ldots, k\}$ otherwise.

Fix $\hat{\Psi} \in \mathcal{V}$, let us to prove that

$$
W^{s}\left(\Gamma_{\Psi}^{c s} ; \hat{\Psi}\right) \cap W^{u}\left(\Gamma_{\Psi}^{c u} ; \hat{\Psi}\right) \neq \emptyset
$$

where $\Gamma_{\Psi}^{c s}, \Gamma_{\Psi}^{c u}$ are the maximal invariant sets in $\Sigma_{d} \times \overline{D_{c s}}$ and $\Sigma_{d} \times \overline{D_{c u}}$ of $\hat{\Psi}$ respectively. Note that $\Gamma_{\Psi}^{c s}$ and $\Gamma_{\Psi}^{c u}$ coincide, respectively, with the maximal invariant set in $\Sigma_{k} \times \overline{D_{c s}}$ and $\Sigma_{k} \times \overline{D_{c u}}$ for $\Psi=\left.\hat{\Psi}\right|_{\Sigma_{k} \times M}$. Hence, from the covering property assumption and Theorem $C$, theses two $\hat{\Psi}$-invariant sets are symbolic blenders for $\Psi$ whose superposition domains contain $B_{c s}$ and $B_{c u}$ respectively.

We observe that in the next lemma we do not use the blender property but rather that $D_{c s}$ $\left(D_{c u}\right)$ are forward (backward) invariant open sets for contractive (expanding) fiber maps of $\Phi$ and its perturbations.

Lemma 5.3. It holds that $C^{+} \times\{y\} \subset W^{s}\left(\Gamma_{\Psi}^{c s} ; \hat{\Psi}\right) \cap W^{u}\left(\Gamma_{\Psi}^{c u} ; \hat{\Psi}\right)$.

Proof. To prove this lemma we need the following claim. Here $\Sigma_{k, k+2}^{+}$represents the bisequences $\xi=\left(\xi_{i}\right)_{i \in \mathbb{Z}} \in \Sigma_{k+2}$ such that $\xi_{i} \in\{1, \ldots, k\}$ for $i \geq 0$, and $\Sigma_{k, k+2}^{-}$the bi-sequences such that $\xi_{i} \in\{1, \ldots, k\}$ for $i<0$.

Claim 5.4. $\Sigma_{k, k+2}^{+} \times D_{c s} \subset W^{s}\left(\Gamma_{\Psi}^{c s} ; \hat{\Psi}\right)$ and $\Sigma_{k, k+2}^{-} \times D_{c u} \subset W^{u}\left(\Gamma_{\Psi}^{c u} ; \hat{\Psi}\right)$.

Proof of the claim. We will prove the first inclusion and the second inclusion is analogous. From Theorem 2.3, the set $\Gamma_{\Psi}^{c s}$ is an attractive graph and so

$$
\lim _{n \rightarrow \infty} d\left(\hat{\Psi}^{n}(\zeta, z), \Gamma_{\Psi}^{c s}\right)=0, \quad \text { for all }(\zeta, z) \in \Sigma_{k} \times D_{c s} .
$$

Thus if $(\xi, p) \in \Sigma_{k, k+2}^{+} \times D_{c s}$ then

$$
\emptyset \neq W_{l o c}^{s s}((\xi, p) ; \hat{\Psi}) \cap\left(\Sigma_{k} \times D_{c s}\right) \subset W^{s}\left(\Gamma_{\Psi} ; \hat{\Psi}\right) .
$$

Now, using the triangular inequality it holds

$$
d\left(\hat{\Psi}^{n}(\xi, p), \Gamma_{\Psi}^{c s}\right) \leq d\left(\hat{\Psi}^{n}(\xi, p), \hat{\Psi}^{n}(\zeta, z)\right)+d\left(\hat{\Psi}^{n}(\zeta, z), \Gamma_{\Psi}^{c s}\right)
$$

where $(\zeta, z) \in W_{l o c}^{s s}((\xi, p) ; \hat{\Psi}) \cap\left(\Sigma_{k} \times D_{c s}\right)$. According to Proposition 2.1 and (5.1) the right part of the above inequality converges to zero and therefore we conclude the claim.

To obtain the lemma we only need to note that $C^{+} \times\{y\} \subset \Sigma_{k, d}^{-} \times D_{c u}$ and thus by the above claim belongs to $W^{u}\left(\Gamma_{\Psi}^{\mathcal{c}} ; \hat{\Psi}\right)$. On the other hand, using that $\hat{\Psi} \in \mathcal{V}$ and again the claim, it follows that

$$
\hat{\Psi}^{m}\left(C^{+} \times\{y\}\right) \subset \Sigma_{k, d}^{+} \times D_{c \mathcal{S}} \subset W^{s}\left(\Gamma_{\Psi}^{c s} ; \hat{\Psi}\right),
$$

which concludes the proof of the lemma.

The next lemma shows the other intersection of the symbolic cycle, namely

$$
W^{u}\left(\Gamma_{\Psi}^{c s} ; \hat{\Psi}\right) \cap W^{s}\left(\Gamma_{\Psi}^{c u} ; \hat{\Psi}\right) \neq \emptyset .
$$

This is achieved by the following stronger result as a consequence of the blender properties. 
Lemma 5.5. It holds that $W^{u u}\left(\Gamma_{\Psi}^{c s} ; \hat{\Psi}\right) \cap W^{s s}\left(\Gamma_{\Psi}^{c u} ; \hat{\Psi}\right) \neq \emptyset$.

Proof. Take the point $x \in B_{c s}$ from the cyclic intersection hypothesis satisfying $\phi_{k+1}^{n}(x) \in B_{c u}$. Since $B_{c u} \subset \mathscr{P}\left(\Gamma_{\Psi}^{c u}\right)$, there exists a bi-sequence $\xi \in \Sigma_{k}$ such that $\left(\xi, \phi_{k+1}^{n}(x)\right) \in \Gamma_{\Psi}^{c u}$.

Consider $\zeta=\left(\zeta_{i}\right)_{i \in \mathbb{Z}} \in W_{\text {loc }}^{s}(\xi ; \tau)$ with $\zeta_{i}=k+1$ for $-n \leq i<0$, and let

$$
z=\gamma_{\xi, \phi_{k+1}^{n}(x), \hat{\Psi}^{S}}^{S}(\zeta)
$$

Remember that $\gamma^{s}$ is the graph map of the local strong stable set, which is itself an almost $\delta$-horizontal disk. Thus, $\left\|z-\phi_{k+1}^{n}(x)\right\|<\delta$ and by the hypothesis on the neighborhood $\mathcal{V}$ of $\hat{\Phi}$ we have that

$$
\hat{\Psi}^{-n}(\zeta, z) \in \Sigma_{k+2} \times B_{c S} .
$$

By the property of the blender (see Proposition 4.7 and Remark 4.9), $W_{l o c}^{s s}\left(\hat{\Psi}^{-n}(\zeta, z) ; \hat{\Psi}\right) \cap$ $W_{l o c}^{u u}\left(\Gamma_{\Psi}^{c s} ; \hat{\Psi}\right) \neq \emptyset$. Let $(\beta, t)$ be a point on this intersection, then it is in $W_{l o c}^{u u}\left(\Gamma_{\Psi}^{c s} ; \hat{\Psi}\right)$ and since $(\beta, t) \in W_{l o c}^{s s}\left(\hat{\Psi}^{-n}(\zeta, z) ; \hat{\Psi}\right)$ then

$$
\hat{\Psi}^{n}(\beta, t) \in W_{l o c}^{s s}((\zeta, z) ; \hat{\Psi})=W_{l o c}^{s s}\left(\left(\xi, \phi_{k+1}^{n_{1}}(x)\right) ; \hat{\Psi}\right) \subset W_{l o c}^{s s}\left(\Gamma_{\Psi}^{c u} ; \hat{\Psi}\right),
$$

proving the lemma.

Combining the above two lemmas the proof of the proposition is now complete.

Now we are ready to prove Theorem A.

Proof of Theorem A. Fix positive constants, $\gamma, \hat{\gamma}, \lambda, \beta$ such that

$$
1 / 2<\gamma \leq \lambda<\beta<1 \leq \hat{\gamma}^{-1}<2 .
$$

We consider an arc of $C^{1}$-diffeomorphisms $\left\{h_{\varepsilon}\right\}_{\varepsilon \in\left[0, \varepsilon_{0}\right]}, h_{\varepsilon}: M \rightarrow M$ such that $h_{0}=$ id and for each $\varepsilon>0$ the map $h_{\varepsilon}$ has two hyperbolic fixed points $p=p(\varepsilon)$ and $q=q(\varepsilon)$ such that $p$ is a $\operatorname{sink}, q$ is a source,

$$
W^{s}\left(p, h_{\varepsilon}\right) \pitchfork W^{u}\left(q, h_{\varepsilon}\right) \neq \emptyset,
$$

and $d(p(\varepsilon), q(\varepsilon)) \rightarrow 0$ as $\varepsilon \rightarrow 0$. We also assume that there are pairwise disjoint neighborhoods $D_{c s}=D_{c s}(\varepsilon)$ and $D_{c u}=D_{c u}(\varepsilon)$ of $p$ and $q$ such that the restrictions $h_{\varepsilon}: \overline{D_{c s}} \rightarrow D_{c s}$ and $h_{\varepsilon}^{-1}: \overline{D_{c u}} \rightarrow D_{c u}$ are $(\lambda, \beta)$-Lipschitz maps.

The next useful lemma tells us how to obtain the covering property from perturbations of a single map. See the proof in [NP12, Prop. 3.2] and [HN11].

Lemma 5.6. Let $\phi_{1}: \bar{D} \rightarrow D$ be a $(\lambda, \beta)$-Lipschitz map with $v^{\alpha}<\lambda<\beta<1$. Then there are a natural number $k$, an open neighborhood $B$ of the fixed point of $\phi_{1}$ and translations (in local coordinates) $\phi_{2}, \ldots, \phi_{k}$ of $\phi_{1}$ such that $\bar{B} \subset \phi_{1}(B) \cup \cdots \cup \phi_{k}(B)$. Consequently, the one-step map

$$
\Phi=\tau \ltimes\left(\phi_{1}, \ldots, \phi_{k}\right) \in \mathcal{S}_{k, \lambda, \beta}^{0, \alpha}(D)
$$

has a symbolic cs-blender whose superposition domain contains $B$. 
We observe that the number $k$ of translations of $\phi_{1}$ depends on the dimension of $M$ and the contraction bound $\lambda$ of $\phi_{1}$.

Going back to the proof of the theorem, by Lemma 5.6 there are $k$ (depends only on $\lambda$ and the dimension of $M$ ) and open sets $B_{c s} \subset D_{c s}, B_{c u} \subset D_{c s}$ containing $p$ and $q$ respectively such that

$$
\overline{B_{c s}} \subset \phi_{1}^{s}\left(B_{c s}\right) \cup \cdots \cup \phi_{k}^{u}\left(B_{c s}\right) \text { and } \overline{B_{c u}} \subset \phi_{1}^{u}\left(B_{c s}\right) \cup \cdots \cup \phi_{k}^{u}\left(B_{c s}\right)
$$

where $\phi_{1}^{s}=h_{\varepsilon}$ on $\overline{D_{c s}}, \phi_{1}^{u}=h_{\varepsilon}^{-1}$ on $\overline{D_{c u}}$ and $\phi_{i}^{s}=\phi_{i}^{s}, \phi_{i}^{u}$ are, respectively, translations of $\phi_{1}^{s}$, $\phi_{1}^{u}$ for all $i=2, \ldots, k$ (which depends on $\varepsilon$ ).

Set $\phi_{1}=h_{\varepsilon}$ and let $\phi_{i}$ be $\left(\gamma, \hat{\gamma}^{-1}\right)$-Lipschitz $C^{1}$-diffeomorphisms on $M$ such that $\phi_{i} \mid \overline{D_{c s}}=\phi_{i}^{s}$ and $\phi_{i} \mid \overline{D_{c u}}=\left(\phi_{i}^{u}\right)^{-1}$ for all $i=2, \ldots, k$. Note that

$$
\overline{B_{c s}} \subset \bigcup_{i=1}^{k} \phi_{i}\left(B_{c s}\right) \text { and } \overline{B_{c u}} \subset \bigcup_{i=1}^{k} \phi_{i}^{-1}\left(B_{c u}\right)
$$

and

$$
\Phi=\tau \ltimes\left(\phi_{1}, \ldots, \phi_{k}\right) \in \mathcal{S}_{k, \lambda_{c s}, \beta_{c s}}^{1, \alpha}\left(D_{c s}\right) \cap \mathcal{S}_{k, \lambda_{c u}, \beta_{c u}}^{1, \alpha}\left(D_{c u}\right)
$$

with $\lambda_{c s}=\lambda, \beta_{c s}=\beta, \lambda_{c u}=\beta^{-1}$ and $\beta_{c u}=\lambda^{-1}$.

Now let us build a transition map between the regions $D_{c s}$ and $D_{c u}$. Fix a small $\varepsilon>0$ and consider the map, in local coordinates, $\varphi(x)=x+q-p$. From the choice of $p$ and $q$, it holds $\varphi=\varphi_{\varepsilon}$ goes to the identity when $\varepsilon \rightarrow 0$.

By hypothesis, $F$ has a Small horseshoe $\Lambda$. We consider a natural number $\ell$ such that $\left.F^{\ell}\right|_{\Lambda}$ is conjugated to the full shift of $d$ symbols with $d \geq k+1$ and we take $R_{1}, \ldots, R_{d}$ rectangles in the ambient manifold $N$ such that $\left\{R_{1} \cap \Lambda, \ldots, R_{d} \cap \Lambda\right\}$ is a Markov partition for $\left.F^{\ell}\right|_{\Lambda}$. Moreover, assume that for every unit vectors $v$ and $w$ in the stable and unstable direction of $\Lambda$ respectively it holds

$$
\mu \leq\left\|D F^{\ell}(v)\right\| \leq v \quad \text { and } \quad \mu \leq\left\|D F^{-\ell}(w)\right\| \leq v
$$

for some positive constant $\mu \leq v \leq v^{\alpha}<1 / 2$ being $\alpha=\log v / \log \mu \in(0,1]$.

We now modify $f_{0}=F \times$ id in $f_{0}^{\ell-1}\left(R_{i} \times M\right)$ to get a one-parameter family of diffeomorphisms $f_{\varepsilon}$ satisfying

$$
\begin{array}{ll}
\left.f_{\varepsilon}^{\ell}\right|_{R_{i} \times M}=F^{\ell} \times \phi_{i} & \text { for } i=1, \ldots, k \\
\left.f_{\varepsilon}^{\ell}\right|_{R_{i} \times M}=F^{\ell} \times \varphi & \text { for } i=k+1, \ldots, d .
\end{array}
$$

Note that the locally constant skew-product diffeomorphism $f_{\varepsilon}^{\ell}$ restricted to the set $\Lambda \times M$ is conjugated to the partially hyperbolic symbolic skew-product

$$
\Psi_{\varepsilon}=\tau \ltimes\left(\phi_{1}, \ldots, \phi_{k}, \varphi, \ldots, \varphi\right) \in \mathcal{P} \mathcal{H} \mathcal{S}_{d}^{1, \alpha}(M) .
$$

In fact, from Proposition A.2, for every small enough $C^{1}$-perturbation $g$ of $f_{\varepsilon}$, the iterate $g^{\ell}$ has an invariant set $\Delta \cong \Lambda \times M$ such that $\left.g^{\ell}\right|_{\Delta}$ is conjugated with a $\mathcal{S}^{1, \alpha}$-perturbation $\Psi_{g^{\ell}}$ of $\Psi_{\varepsilon}$. 
Observe that $\Psi_{\varepsilon}$ satisfies the assumptions in Proposition 5.2: the maps $\phi_{i}$ have the the covering properties in the regions $B_{c s}, B_{c u}$, and the transition maps between these domains are created via the map $\varphi$ and using the hypothesis that $W^{s}\left(p, h_{\varepsilon}\right) \pitchfork W^{u}\left(q, h_{\varepsilon}\right) \neq \emptyset$. Then the symbolic skew-product $\Psi_{\varepsilon}$ has a $\mathcal{S}^{1, \alpha}$-robust symbolic cycle associated with symbolic blenders.

Hence, according to Proposition A.2, using the conjugation, $f_{\varepsilon}^{\ell}$ has a $C^{1}$-robust cycle associated with hyperbolic sets $\Gamma_{\varepsilon}^{c s}$ and $\Gamma_{\varepsilon}^{c u}$ for $f_{\varepsilon}^{\ell}$ that come from the symbolic blenders. To compute the co-index of this cycle note that the (stable) indices of $\Gamma_{\varepsilon}^{c s}$ and $\Gamma_{\varepsilon}^{c u}$ are, respectively, $\operatorname{Ind}(\Lambda)+\operatorname{dim}(M)$ and $\operatorname{Ind}(\Lambda)$. Thus, the co-index of the heterodimensional cycle is equal to the dimension of $M$.

The one-parameter family may be taken so that the same conclusions of a heterodimensional cycle with the above co-index between two hyperbolic sets can be made for the map $f_{\varepsilon}$. This ends the proof of the theorem.

5.2. Robust mixing: proof of Theorem B. We will build robustly mixing examples of symbolic skew-products. One of the classical ways to create robustly transitive diffeomorphisms is to construct a map that robustly has a hyperbolic periodic point with dense stable and unstable manifolds. Hence, using the inclination lemma (or $\lambda$-lemma) one concludes the the diffeomorphism is robustly topologically mixing. We will do the same here for the symbolic case in the following theorem.

Theorem 5.7. Let $\hat{\Phi} \in \mathcal{P} \mathcal{H} \mathcal{S}_{d}^{1, \alpha}(M)$ be a one-step map with $d>k$. Assume that the restriction

$$
\Phi=\left.\hat{\Phi}\right|_{\Sigma_{k} \times M}=\tau \ltimes\left(\phi_{1}, \ldots, \phi_{k}\right)
$$

has a symbolic cs-blender $\Gamma_{\Phi}^{c s} \subset \Sigma_{k} \times M$ whose superposition domain contains an open set $B \subset M$ satisfying that $\bar{B} \subset \phi_{1}(B) \cup \cdots \cup \phi_{k}(B)$.

Assume that there exists an attracting fixed point of $\phi_{1}$ and a repelling fixed point of $\phi_{k+1}$, both in $B$, having respectively stable and unstable manifold $C^{1}$-robustly dense in $M$.

Then, there exists a fixed point $(\vartheta, p) \in \Gamma_{\Phi}^{c s}$ for $\hat{\Phi}$ such that its stable and unstable sets,

$$
W^{s}((\vartheta, p) ; \hat{\Phi}) \text { and } W^{u}((\vartheta, p) ; \hat{\Phi}) \text {, }
$$

are $\mathcal{S}^{1, \alpha}$-robustly dense on $\Sigma_{d} \times M$. Consequently $\hat{\Phi}$ is $\mathcal{S}^{1, \alpha}$-robustly topologically mixing.

The next sequence of propositions are necessarily to prove the above theorem. The first proposition shows how to enlarge the topological dimension of the closure of the strong unstable set of a blender via a fiber-repelling fixed point. Compare to [BDV05, Lemma 6.12].

Proposition 5.8 (Blender activation). Let $\hat{\Phi} \in \mathcal{P} \mathcal{H} \mathcal{S}_{d}^{0, \alpha}(M)$ be a one-step map in the hypothesis of Proposition 4.7. Assume that there exists a fiber-repelling fixed point $(v, q)$ of $\hat{\Phi}$ in $\Sigma_{d} \times B$. Then for every small enough $\mathcal{S}^{0, \alpha}$-perturbation $\hat{\Psi}$ of $\hat{\Phi}$ it holds that

$$
W^{u}\left(\left(v, q_{\hat{\Psi}}\right) ; \hat{\Psi}\right) \subset \overline{W^{u u}\left(\Gamma_{\Psi}^{c s} ; \hat{\Psi}\right)}
$$

where $\Gamma_{\Psi}^{c s}$ is continuation of $\Gamma_{\Phi}^{c s}$ for $\Psi=\left.\hat{\Psi}\right|_{\Sigma_{k} \times M}$. 
Proof. Assume that $\hat{\Phi}$ satisfies the global domination $v^{\alpha}<\lambda<1<\beta<v^{-\alpha}$. Consider a small $\mathcal{S}^{0, \alpha}$-neighborhood $\mathcal{V}$ of $\hat{\Phi}$ such that for every $\hat{\Psi} \in \mathcal{V} C v^{\alpha}<\delta$ where $C=C_{\Psi}\left(1-\lambda^{-1} v^{\alpha}\right)^{-1}$ and $\delta>0$ is given in Proposition 4.7. Moreover, we can take $\delta$ small enough such that $B_{2 \delta}(q) \subset B$.

Let $\hat{\Psi} \in \mathcal{V}$ and consider $(\xi, x) \in W^{u}\left(\left(v, q_{\hat{\Psi}}\right) ; \hat{\Psi}\right)$. Let $V$ be an open neighborhood of $(\xi, x)$. In order to prove the proposition we need to show that the global strong unstable set of $\Gamma_{\Psi}^{C S}$ meets $V$. Since $\Psi^{-n}(\xi, x)$ converges to $\left(v, q_{\hat{\Psi}}\right) \in \Sigma_{d} \times B$ then for $n$ large enough we have that $B_{\delta}\left(\Psi^{-n}(\xi, x)\right) \subset \Sigma_{k} \times B$. Now, we define

$$
h_{n}: W_{l o c}^{s}\left(\tau^{-n}(\xi) ; \tau\right) \rightarrow M, \quad h_{n}(\zeta)=\mathscr{P} \circ \hat{\Psi}^{-n}\left(\tau^{n}(\zeta), x\right)=\psi_{\tau^{n-1}(\zeta)}^{-n}(x) .
$$

Let $H_{n}$ be the graph set of $h_{n}$ and notice that for $n$ large

$$
H_{n} \subset \Psi^{-n}\left(\left(W_{l o c}^{s}(\xi ; \tau) \times\{x\}\right) \cap V\right) .
$$

Claim 5.9. For $n$ large enough, $H_{n}$ is a $\delta$-horizontal disk in $\Sigma_{d} \times B$.

Proof. Let $\zeta, \zeta^{\prime} \in W_{l o c}^{s}\left(\tau^{-n}(\xi) ; \tau\right)$ be two bisequences with $d_{\Sigma_{d}}\left(\zeta, \zeta^{\prime}\right)=v^{l}$. Applying Lemma 4.2 to $\tau^{n}(\zeta), \tau^{n}\left(\zeta^{\prime}\right)$ and the point $x$, we obtain

$$
\begin{aligned}
\left\|h_{n}(\zeta)-h_{n}\left(\zeta^{\prime}\right)\right\| & =\left\|\psi_{\tau^{-1}\left(\tau^{n}(\zeta)\right)}^{-n}(x)-\psi_{\tau^{-1}\left(\tau^{n}\left(\zeta^{\prime}\right)\right)}^{-n}(x)\right\| \\
& \leq C \Psi_{\Psi} v^{\alpha((n+l)-n)} \sum_{j=0}^{n-1}\left(\lambda^{-1} v^{\alpha}\right)^{j} \\
& <v^{\alpha l} C_{\mathcal{\Psi}^{\prime}}\left(1-\lambda^{-1} v^{\alpha}\right)^{-1}=C d_{\Sigma_{d}}\left(\zeta, \zeta^{\prime}\right)^{\alpha} .
\end{aligned}
$$

Hence, from the choice of $\delta$ we have proved that $h_{n}$ is $(\alpha, C)$-Hlder continuous map with $C v^{\alpha}<\delta$. On the other hand, for $n$ large enough $H_{n} \subset \Sigma_{d} \times B$ and therefore we conclude the proof of the claim.

From the above claim and Proposition 4.7, for $n$ large enough,

$$
W_{l o c}^{u u}\left(\Gamma_{\Psi}^{c s} ; \hat{\Psi}\right) \cap H_{n} \neq \emptyset \text {. }
$$

This implies that

$$
\emptyset \neq W^{u u}\left(\Gamma_{\Psi}^{c s} ; \hat{\Psi}\right) \cap \Psi^{n}\left(H_{n}\right) \subset W^{u u}\left(\Gamma_{\Psi}^{c s} ; \hat{\Psi}\right) \cap\left(W_{l o c}^{s}(\xi ; \tau) \times\{x\}\right) \cap V
$$

and completes the proof of the proposition.

The following lemma shows how to construct a one-step map having fixed points with the stable or unstable set robustly dense.

Lemma 5.10. Let $\Phi=\tau \ltimes \phi_{\xi} \in \mathcal{P} \mathcal{H} \mathcal{S}_{d}^{1, \alpha}(M)$ be a one-step skew-product map with a fiberrepelling/attracting fixed point $(v, q)$ such that the unstable/stable manifold of $q$ for $\phi_{v}$ is $C^{1}$-robustly dense in $M$. Then the unstable/stable set of $(v, q)$ for $\Phi$ is $\mathcal{S}^{1, \alpha}$-robustly dense in $\Sigma_{d} \times M$.

Proof. Consider a $\mathcal{S}^{1, \alpha}$-perturbation $\hat{\Psi}=\tau \ltimes \psi_{\xi}$ of $\hat{\Phi}$. We will prove that the unstable set of the fiber-repelling fixed point $\left(v, q_{\hat{\Psi}}\right)$, continuation of $(v, p)$ for $\hat{\Psi}$, is dense in $\Sigma_{d} \times M$. Similarly follows for the case of the fiber-attracting fixed point and its stable set. 
Let $C \times V$ be any basic open set in $\Sigma_{d} \times M$. By the density of the unstable set of $v$ for $\tau$ on $\Sigma_{d}$, there exists $\xi \in C \cap W^{u}(v ; \tau)$ and so for $n$ large enough $\tau^{-n}(\xi)$ is close to $v$. For the fiber maps $\psi_{\zeta}$ with $\zeta$ close enough to $v$, there exists the fiber-repelling fixed point $q_{\hat{\Psi}, \zeta}$, which is the continuation of $q_{\hat{\Psi}}$.

Let $\tilde{V}$ be the closure of a non-empty open set in $V$. Fixing $n$, observe that for every $m \geq 1$ it holds that

$$
\psi_{\tau^{-1}(\xi)}^{-m-n}(\tilde{V})=\psi_{\tau^{-m}\left(\tau^{-n}(\xi)\right)}^{-1} \circ \cdots \circ \psi_{\tau^{-1}\left(\tau^{-n}(\xi)\right)}^{-1} \circ \psi_{\tau^{-1}(\xi)}^{-n}(\tilde{V})
$$

where $\psi_{\tau^{-i}\left(\tau^{-n}(\xi)\right)}$ is $C^{1}$-close to $\psi_{v}$ and thus also to $\phi_{v}$. Since by hypothesis the unstable manifold of $q$ for $\phi_{v}$ is $C^{1}$-robustly dense in $M$, the same holds for the fixed point $q_{\hat{\Psi}, \tau^{-i}\left(\tau^{-n}(\xi)\right)}$ with respect to the maps $\psi_{\tau^{-i}\left(\tau^{-n}(\xi)\right)}$.

By the continuous dependence of compact pieces of the unstable manifold with respect to the fiber diffeomorphisms, we may assume that for all $m \geq 1$, the unstable manifold of $q_{\hat{\Psi}, \tau^{-m}\left(\tau^{-n}(\xi)\right)}$ intersects $\psi_{\tau^{-1}(\xi)}^{-n}(\tilde{V})$.

Thus for each $m \geq 1$ there is the point $x_{m}$ which belongs to the intersection

$$
\psi_{\tau^{-1}(\xi)}^{-n}(\tilde{V}) \cap W^{u}\left(q_{\hat{\Psi}, \tau^{-m}\left(\tau^{-n}(\xi)\right)} ; \psi_{\tau^{-m}\left(\tau^{-n}(\xi)\right)}\right) .
$$

Since $\tilde{V}$ is closed we may assume that the sequence $\left\{x_{m}\right\}$ converges to a point $x$ in $\psi_{\tau^{-1}(\xi)}^{-n}(\tilde{V}) \subset$ $\psi_{\tau^{-1}(\xi)}^{-n}(V)$. By the Hölder continuity of fiber maps on the base sequence, $q_{\hat{\Psi}, \tau^{-m}\left(\tau^{-n}(\xi)\right)}$ tends to $q_{\Psi}$, and then it is not hard to show that $\hat{\Psi}^{-m-n}\left(\tau^{-n}(\xi), x\right)$ goes to $\left(v, q_{\hat{\Psi}}\right)$ as $m \rightarrow \infty$. Thus

$$
\hat{\Psi}^{n}\left(\tau^{-n}(\xi), x\right) \in(C \times V) \cap W^{u}\left(\left(v, q_{\hat{\Psi}}\right) ; \hat{\Psi}\right),
$$

concluding the proof of the lemma.

The following proposition demonstrates how to create a symbolic cs-blender with a dense strong unstable set.

Proposition 5.11. Let $\hat{\Phi} \in \mathcal{P} \mathcal{H} \mathcal{S}_{d}^{1, \alpha}(M)$ be a one-step in the hypothesis of Proposition 4.7 with $d>k$. Assume that the fiber map $\phi_{k+1}$ has a repelling fixed point in $B$ with the unstable manifold $C^{1}$-robustly dense on $M$. Then for every small enough $\mathcal{S}^{1, \alpha}$-perturbation $\hat{\Psi}$ of $\hat{\Phi}$ it holds that

$$
\overline{W^{u u}\left(\Gamma_{\Psi}^{c s} ; \hat{\Psi}\right)}=\Sigma_{d} \times M
$$

where $\Gamma_{\Psi}^{c s}$ is the continuation of $\Gamma_{\Phi}^{c s}$ for $\Psi=\left.\hat{\Psi}\right|_{\Sigma_{k} \times M}$.

Proof. Let $q \in B$ be the repelling fixed point of $\phi_{k+1}$. Observe that $(v, q)$ is a fiber-repelling fixed point of $\hat{\Phi}$ in $\Sigma_{d} \times B$ where $v=\left(v_{i}\right)_{i \in \mathbb{Z}} \in \Sigma_{d}$ is the bi-sequence with $v_{i}=k+1$ for all $i \in \mathbb{Z}$. By Proposition 5.8 it follows that for every small enough $\mathcal{S}^{1, \alpha}$-perturbation $\hat{\Psi}$ of $\hat{\Phi}$,

$$
W^{u}\left(\left(v, q_{\hat{\Psi}}\right) ; \hat{\Psi}\right) \subset \overline{W^{u u}\left(\Gamma_{\Psi}^{c s} ; \hat{\Psi}\right)}
$$

By Lemma 5.10, $W^{u}\left(\left(v, q_{\hat{\Psi}}\right) ; \hat{\Psi}\right)$ is dense in $\Sigma_{d} \times M$ implying the density of the strong unstable set of $\Gamma_{\hat{\Psi}}^{c S}$ for $\hat{\Psi}$. 
Lemma 5.12. Consider a symbolic skew-product $\hat{\Phi} \in \mathcal{P} \mathcal{H} \mathcal{S}_{d}^{0, \alpha}(M)$ and let $\Gamma \subset \Sigma_{d} \times M$ be a $\hat{\Phi}$ invariant set such that

$$
\Gamma \subset \overline{W^{u u}((\vartheta, p) ; \hat{\Phi})}
$$

where $(\vartheta, p)$ is a fixed point of $\hat{\Phi}$. Then $W^{u u}(\Gamma ; \hat{\Phi}) \subset \overline{W^{u u}((\vartheta, p) ; \hat{\Phi})}$.

Proof. Consider $(\xi, x) \in W^{u u}(\Gamma ; \hat{\Phi})$ and let $V$ be an open neighborhood of $(\xi, x)$. In order to prove the lemma we need to show that the strong unstable set of the fixed point $(\vartheta, p)$ meets $V$. Since $(\xi, x)$ belongs to the global strong unstable set of $\Gamma$ and $\Gamma$ is $\hat{\Phi}$-invariant set then there are $n \in \mathbb{N}$ and $(\zeta, z) \in \Gamma$ such that $\hat{\Phi}^{-n}(\xi, x) \in W_{l o c}^{u u}((\zeta, z) ; \hat{\Phi})$. Hence

$$
\hat{\Phi}^{-n}(V) \cap W_{l o c}^{u u}((\zeta, z) ; \hat{\Phi}) \neq \emptyset .
$$

Since the global strong unstable set of $(\vartheta, p)$ lies densely in $\Gamma$ then there exists a sequence $\left(\left(\xi^{(k)}, x^{(k)}\right)\right)_{k} \subset W^{u u}((\vartheta, p) ; \hat{\Phi})$ converging to $(\zeta, z)$.

According to Proposition 2.1, the local strong unstable set $W_{l o c}^{u u}((\xi, x) ; \hat{\Phi})$ varies continuously with respect to $(\xi, x)$ and so $W_{l o c}^{u u}\left(\left(\xi^{(k)}, x^{(k)}\right) ; \hat{\Phi}\right)$ converges to $W_{l o c}^{u u}((\zeta, z) ; \hat{\Phi})$. Since $\hat{\Phi}^{-n}(V)$ is an open set in $\Sigma_{d} \times M$, then this convergence and (5.2) imply that for $k$ large enough

$$
\hat{\Phi}^{-n}(V) \cap W_{l o c}^{u u}\left(\left(\xi^{(k)}, x^{(k)}\right) ; \hat{\Phi}\right) \neq \emptyset .
$$

Therefore,

$$
\emptyset \neq V \cap \hat{\Phi}^{n}\left(W_{l o c}^{u u}\left(\left(\xi^{(k)}, x^{(k)}\right) ; \hat{\Phi}\right)\right) \subset V \cap W^{u u}((\vartheta, p) ; \hat{\Phi}),
$$

completing the proof of the lemma.

Now, we are ready to give the proof of Theorem 5.7.

Proof of Theorem 5.7. Let us denote by $(\vartheta, p)$ and $(v, q)$, respectively, the fiber-attracting and fiber-repelling fixed points of $\hat{\Phi}$ in $\Sigma_{d} \times B$ where the bi-sequences $\vartheta=\left(\vartheta_{i}\right)_{i \in \mathbb{Z}}$ and $v=\left(v_{i}\right)_{i \in \mathbb{Z}}$ are given by $\vartheta_{i}=1, v_{i}=k+1$ for all $i \in \mathbb{Z}$. Observe that $(\vartheta, p)$ belongs to $\Gamma_{\Phi}^{c S}$.

Since by hypothesis the stable manifold of $p$ for $\phi_{1}$ is $C^{1}$-robustly dense on $M$, then we can apply Lemma 5.10 to conclude that the stable set of $(\vartheta, p)$ for $\hat{\Phi}$ is $\mathcal{S}^{1, \alpha}$-robustly dense on $\Sigma_{d} \times M$.

Moreover, from Theorem 2.3, (see also Proposition B.6), it holds that the strong unstable set lies $\mathcal{S}^{1, \alpha}$-robustly dense in $\Gamma_{\Phi}^{c s}$. Therefore, by Lemma 5.12 it follows that

$$
W^{u u}\left(\Gamma_{\Phi}^{c s} ; \hat{\Phi}\right) \subset \overline{W^{u}((\vartheta, p) ; \hat{\Phi})}
$$

in a $\mathcal{S}^{1, \alpha}$-robust sense.

By Proposition 5.11, the strong unstable set of $\Gamma_{\Phi}^{c s}$ for $\hat{\Phi}$ is $\mathcal{S}^{1, \alpha}$-robustly dense on $\Sigma_{d} \times M$ and thus we obtain that the unstable manifold of $(\vartheta, p)$ also lies $\mathcal{S}^{1, \alpha}$-robustly dense in $\Sigma_{d} \times M$.

Therefore both the stable and unstable sets of $(\vartheta, p)$ are $\mathcal{S}^{1, \alpha}$-robustly dense in $\Sigma_{d} \times M$.

In order to conclude the proof of the theorem we need to prove that $\hat{\Phi}$ is $\mathcal{S}^{1, \alpha}$-robustly topologically mixing. Let $\hat{\Psi}=\tau \ltimes \psi_{\xi}$ be a perturbation of $\hat{\Phi}$ and $\left(\vartheta, p_{\hat{\Psi}}\right)$ the continuation of 
the fixed point $(\vartheta, p)$. Consider a pair of open sets $\hat{U}$ and $\hat{V}$ in $\Sigma_{d} \times M$. By the density of the stable and unstable sets of $\left(\vartheta, p_{\hat{\Psi}}\right)$ for $\hat{\Psi}$, we can take two points

$$
(\eta, y) \in W^{s}\left(\left(\vartheta, p_{\hat{\Psi}}\right) ; \hat{\Psi}\right) \cap \hat{U} \quad \text { and } \quad(\zeta, z) \in W^{u}\left(\left(\vartheta, p_{\hat{\Psi}}\right) ; \hat{\Psi}\right) \cap \hat{V} .
$$

Fix $\varepsilon>2 \epsilon>0$ small enough and assume that $\hat{\Psi}$ belongs to a small perturbative neighborhood of $\hat{\Phi}$ such that $C_{\hat{\Psi}}\left(1-\lambda^{-1} v^{\alpha}\right)^{-1}<\epsilon$ and

$$
W_{l o c}^{u u}((\xi, x) ; \hat{\Psi}) \subset \Sigma_{d} \times B_{\varepsilon}\left(p_{\hat{\Psi}}\right) \quad \text { for all }(\xi, x) \in \Sigma_{d} \times B_{\epsilon}\left(p_{\hat{\Psi}}\right) .
$$

Since $(\zeta, z)$ belongs to the unstable set of $\left(\vartheta, p_{\hat{\Psi}}\right)$ and by Lemma 4.2 , it follows that for every $n$ large enough and $\xi \in W_{l o c}^{s}(\zeta ; \tau)$ with $d_{\Sigma_{d}}(\xi, \zeta) \leq v^{n}$ we have that

$$
\begin{aligned}
\left\|\psi_{\tau^{-1}(\xi)}^{-n}(z)-p_{\hat{\Psi}}\right\| & \leq\left\|\psi_{\tau^{-1}(\xi)}^{-n}(z)-\psi_{\tau^{-1}(\zeta)}^{-n}(z)\right\|+\left\|\psi_{\tau^{-1}(\zeta)}^{-n}(z)-p_{\hat{\Psi}}\right\| \\
& \leq C_{\hat{\Psi}}\left(1-\lambda^{-1} v^{\alpha}\right)^{-1}+\epsilon<2 \epsilon<\varepsilon .
\end{aligned}
$$

By continuity, we can take an open set $V$ in $M$ and a cylinder $C$ in $\Sigma_{d}$ around of $\zeta$ of large enough length $n$ such that $C \times V \subset \hat{V}$ and

$$
W_{l o c}^{s}\left(\tau^{-n}(\zeta) ; \tau\right) \times B_{\varepsilon}\left(p_{\hat{\Psi}}\right) \subset \hat{\Psi}^{-n}\left(\left(W_{l o c}^{s}(\zeta ; \tau) \cap C\right) \times V\right) \subset \hat{\Psi}^{-n}(\hat{V}) .
$$

On the other hand, since $(\eta, y)$ belongs to the stable set of $\left(\vartheta, p_{\hat{\Psi}}\right)$ it follows that $\psi_{\eta}^{m}(y) \in$ $B_{\epsilon}\left(p_{\hat{\Psi}}\right)$ for all $m$ large enough. Hence, (5.3) implies that for every $m$ large enough

$$
\begin{aligned}
\emptyset & \neq W_{l o c}^{u u}(\hat{\Psi}(\eta, y) ; \hat{\Psi}) \cap\left(W_{l o c}^{s}\left(\tau^{-n}(\zeta) ; \tau\right) \times B_{\varepsilon}\left(p_{\hat{\Psi}}\right)\right) \\
& \subset \hat{\Psi}^{m}\left(W_{l o c}^{u u}((\eta, y) ; \hat{\Psi}) \cap \hat{U}\right) \cap \hat{\Psi}^{-n}(\hat{V}) .
\end{aligned}
$$

Therefore, for all $n$ and $m$ large enough $\hat{\Psi}^{n+m}(\hat{U}) \cap \hat{V} \neq \emptyset$, proving that $\hat{\Psi}$ is topologically mixing.

Observe that diffeomorphisms that have fixed points with robustly dense stable/unstable manifolds can be constructed on any manifold $M$ by perturbations of time-one maps of gradient-like vector fields. This completes our objective in building robustly mixing examples in the set of symbolic skew-products (compare to [Hom12, Lemma 4.1] and the application in [HN11]). Lastly we conjugate these examples with partially hyperbolic sets on manifolds.

Proof of Theorem $B$. The construction of an arc of diffeomorphisms on $N \times M$ with robustly topologically mixing non-hyperbolic sets is similar to the proof of Theorem A. Thus we will just note the main differences.

As in Theorem A, we modify $f_{0}=F \times$ id to get a one-parameter family of diffeomorphisms $f_{\varepsilon}$ satisfying

$$
\begin{array}{ll}
\left.f_{\varepsilon}^{\ell}\right|_{R_{i} \times M}=F^{\ell} \times \phi_{i} & \text { for } i=1, \ldots, k \\
\left.f_{\varepsilon}^{\ell}\right|_{R_{i} \times M}=F^{\ell} \times \varphi & \text { for } i=k+1, \ldots, d
\end{array}
$$

The maps $\phi_{i}=\phi_{i, \varepsilon}$ for $i=2, \ldots, k$ are obtained from Lemma 5.6 as small translations (on local coordinates) of a $C^{1}$-perturbation $\phi_{1}=\phi_{1, \varepsilon}$ of the identity map with an attracting fixed point $p$ with unstable manifold $C^{1}$-robustly dense in $M$. 
Moreover, the the iterated function system generated by $\phi_{1}, \ldots, \phi_{k}$ satisfies the covering property in an open set $B_{c s}$ with $p \in B_{c s}$. The map $\varphi=\varphi_{\varepsilon}$ is a $C^{1}$-perturbation of id with a repelling fixed point in $B_{c S}$ whose unstable manifold is $C^{1}$-robustly dense on $M$.

Notice that the restriction of $f_{\varepsilon}^{\ell}$ to $\Lambda \times M$ is conjugated to

$$
\Phi_{\varepsilon}=\tau \ltimes\left(\phi_{1}, \ldots, \phi_{k}, \varphi, \ldots, \varphi\right) \in \mathcal{P} \mathcal{H} \mathcal{S}_{d}^{1, \alpha}(M) .
$$

This partially hyperbolic one-step satisfies the assumptions in Theorem 5.7 and thus $\Phi_{\varepsilon}$ is $\mathcal{S}^{1, \alpha}$-robustly topologically mixing. By Proposition A.2, via the conjugation, it follows that $\left.f_{\varepsilon}^{\ell}\right|_{\Lambda \times M}$ is $C^{1}$-robustly topologically mixing and thus the same holds for $\left.f_{\varepsilon}\right|_{\Lambda \times M}$.

To show that $\Lambda \times M$ and its continuations $\Delta_{g}$ (for small $C^{1}$-perturbation $g$ of $f_{\varepsilon}$ ) are non-hyperbolic sets, let us observe that the map $\Phi_{\varepsilon}$ by construction has fiber-repelling and fiber-attracting fixed points. Therefore, via the conjugation, the topologically mixing sets $\Delta_{g}$ on the manifold $N \times M$ will have periodic points of (stable) index $\operatorname{Ind}(\Lambda)+\operatorname{dim}(M)$ and of index $\operatorname{Ind}(\Lambda)$, thus proving the non-hyperbolicity of these sets.

This completes the proof of the last theorem.

\section{Appendix A. Reduction to Perturbations of Symbolic SKeW-Products}

In this appendix we will explain how to go from partially hyperbolic diffeomorphisms to symbolic skew-products.

Let $F$ be a $C^{2}$-diffeomorphism of $N$ with a horseshoe $\Lambda \subset N$ of $d$-legs which has a DFinvariant splitting of the tangent bundle

$$
T_{\Lambda} N=E_{\Lambda}^{S} \oplus E_{\Lambda}^{u}
$$

such that in a Riemannian metric on $N$ there is $0<\mu<v<1$ satisfying

$$
\mu \leq\left\|D_{z} F(v)\right\| \leq v \quad \text { and } \quad \mu \leq\left\|D_{z} F^{-1}(w)\right\| \leq v
$$

for all unit vectors $v \in E_{z}^{s}, w \in E_{z}^{u}$ and $z \in \Lambda$. We assume that $E_{\Lambda^{\prime}}^{s} E_{\Lambda}^{u}$ are trivial product bundles. Both, the above assumption and the extra regularity of $F$, are technical conditions necessary for the main result in [IN12], but it is conjectured that it holds without them (see also [Gor06, PSW12]). This result is our main tool to prove the next proposition (see Proposition A.2).

Let $f$ be a $C^{1}$ skew-product diffeomorphism over $F$ defined as

$$
f: N \times M \rightarrow N \times M, \quad f(z, x)=(F(z), \phi(z, x))
$$

where $\phi(z, \cdot): M \rightarrow M$ is a family of $C^{1}$-diffeomorphisms such that there are positive numbers $\gamma$ and $\hat{\gamma}$ satisfying

$$
\gamma<\left\|D_{z} \phi(z, \cdot) v\right\|<\hat{\gamma}^{-1} \quad \text { for all unit vector } v \in T_{z} M \text { and } z \in \Lambda \text {. }
$$

We will assume that the skew-product diffeomorphism (A.1) satisfies that

$$
v+L_{f}<v^{-1} \text { and } v<\gamma<\hat{\gamma}^{-1}<v^{-1}
$$


where for a fixed $\delta>0$ small enough

$$
L_{f}=\sup _{x \in M}\left\{\frac{\left\|\phi^{ \pm 1}(z, x)-\phi^{ \pm 1}\left(z^{\prime}, x\right)\right\|}{d_{N}\left(z, z^{\prime}\right)}: z, z^{\prime} \in \Lambda, 0<d_{N}\left(z, z^{\prime}\right)<\delta\right\} \geq 0 .
$$

We say that $L_{f}$ is the local Lipschitz constant of $f\left(\right.$ or $\left.f^{-1}\right)$. The inequalities (A.3) are called modified dominated splitting condition in [IN12]. The first inequality is clearly verified if $L_{f}=0$. The other inequalities are the dominated conditions in the definition of partial hyperbolicity.

Definition A.1. A skew-product diffeomorphism $f$ as (A.1) is said to be locally constant if $L_{f}=0$.

Partial hyperbolic locally constant skew-product diffeomorphisms over a horseshoe satisfy the modified dominated splitting condition (A.3). Modified dominated splitting condition is a $C^{1}$-open condition since the same property is satisfied for any diffeomorphism $C^{1}$ close. However, a $C^{1}$-close diffeomorphism $g$ of $f$ is a priori not a skew-product. Under the assumptions (A.3), from Hirsch-Pugh-Shub theory [HPS77] or from the recent works of [Gor06, IN12, PSW12] it follows that $g$ is topologically conjugated to a skew-product. We will explain more about this.

Proposition A.2. Let $f$ be a $C^{1}$-skew-product diffeomorphism as (A.1) satisfying (A.2) and (A.3). Then given $\varepsilon>0$ small enough, any $\varepsilon$-perturbation $g$ of $f$ in the $C^{1}$-topology has a locally maximal invariant set $\Delta \subset N \times M$ homeomorphic to $\Lambda \times M$ such that $\left.g\right|_{\Delta}$ is conjugated to a symbolic skew-product

$$
\Psi_{g} \in \mathcal{P} \mathcal{H} \mathcal{S}_{d}^{1, \alpha}(M)
$$

with $\alpha=\log v / \log \mu>0$ and local Hölder constant $C_{\Psi_{g}}=L_{f}+O(\varepsilon)$.

Remark A.3. In the special case of $f=F \times$ id, from [Gor06, Theorem B], the above Proposition A.2 holds without both technical assumptions, the extra regularity of the base map $F$ and the trivialization of the vector bundles.

Proof of Proposition A.2. For each $z \in \Lambda$ we consider the fiber $\mathcal{L}_{z}=\{z\} \times M$. The collection $\mathcal{L}$ of these fibers is an invariant lamination of $f$. In [HPS77, Theorems 7.1] and also in [IN12, Theorem A] and [PSW12, Theorem C] it is showed that this lamination is $C^{1}$-persistent. The $C^{1}$-persistence of such lamination means that for any $C^{1}$-perturbation of $f$, there exists a lamination, $C^{1}$-close to $\mathcal{L}$, which is preserved by the new dynamics, and such that the dynamics induced on the space of the leaves remains the same.

Given $\varepsilon>0$ small enough we take $g$ a $C^{1}$-diffeomorphism $\varepsilon$-close to $f$ in the $C^{1}$-topology. Note that,

$$
g(z, x)=(\tilde{F}(z, x), \tilde{\phi}(z, x)), \quad(z, x) \in N \times M
$$

where $\tilde{\phi}(z, \cdot): M \rightarrow M$ is also a $C^{1}$-diffeomorphism such that

$$
\gamma\left\|x-x^{\prime}\right\|<\left\|\tilde{\phi}(z, x)-\tilde{\phi}\left(z, x^{\prime}\right)\right\|<\hat{\gamma}^{-1}\left\|z-x^{\prime}\right\|
$$

for all $x, x^{\prime} \in M$ and $z$ in a neighborhood of $\Lambda$. 
For each $z \in \Lambda$, the fiber $\mathcal{W}_{\sigma(z)}$, continuation of $\mathcal{L}_{z}$ for $g$ is parametrized by the graph of a $C^{1}$-map $Q(z, \cdot): M \rightarrow M \times N$. According to [IN12, Theorem A] and since $g$ is $\varepsilon$-close to $f$, we get

$$
\begin{aligned}
d_{N}\left(Q(z, x), Q\left(z, x^{\prime}\right)\right) & \leq O(\varepsilon)\left\|x-x^{\prime}\right\|, \\
d_{C^{0}}(Q(z, \cdot), z) & \leq O(\varepsilon) .
\end{aligned}
$$

For $C^{1}$ maps, the $C^{0}$ norm of the first derivative is equal the best Lipschitz constant. Hence, the above inequalities show that $d_{C^{1}}(Q(z, \cdot), z) \leq O(\varepsilon)$.

Let

$$
\Delta=\bigcup_{z \in \Lambda} W_{\sigma(z)} \subset M \times N .
$$

By sending $\mathcal{W}_{\sigma(z)}$ to $z$ we may define a continuous projection $P: \Delta \rightarrow N$ such that $P(\Delta)=\Lambda$ and $\left.F\right|_{\Lambda} \circ P=P \circ g$. Moreover,

$$
h: \Delta \rightarrow \Lambda \times M, \quad h(z, x)=(P(z, x), x)
$$

is an homeomorphism whose inverse is $h^{-1}(z, x)=(Q(z, x), x)$. Let

$$
\tilde{g}=\left.h \circ g\right|_{\Delta} \circ h^{-1}: \Lambda \times M \rightarrow \Lambda \times M .
$$

Observe that

$$
\begin{aligned}
\tilde{g}(z, x) & =\left(P \circ g \circ h^{-1}(z, x), \tilde{\phi} \circ h^{-1}(z, x)\right) \\
& =\left(\left.F\right|_{\Lambda} \circ P \circ h^{-1}(z, x), \tilde{\phi}(Q(z, x), x)\right)=(F(z), \psi(z, x)) .
\end{aligned}
$$

Thus, $\tilde{g}$ is a skew-product diffeomorphism defined on $\Lambda \times M$ which is conjugated to $\left.g\right|_{\Delta}$ by means of the conjugation $h$. Since for each $z \in \Lambda$ the map $\psi(z, \cdot)$ is a composition of $C^{1}$-maps, then it is a $C^{1}$-map. In addition, we can easily check that the rate of contraction and expansion of these maps are uniformly close to $\hat{\gamma}^{-1}$ and $\gamma$ respectively. Indeed,

$$
\begin{aligned}
& \left\|\psi(z, x)-\psi\left(z, x^{\prime}\right)\right\| \leq \\
& \quad \leq\left\|\tilde{\phi}(Q(z, x), x)-\tilde{\phi}\left(Q(z, x), x^{\prime}\right)\right\|+\left\|\tilde{\phi}\left(Q(z, x), x^{\prime}\right)-\tilde{\phi}\left(Q\left(z, x^{\prime}\right), x^{\prime}\right)\right\| .
\end{aligned}
$$

By means of (A.4) and (A.5) it follows that

$$
\left\|\psi(z, x)-\psi\left(z, x^{\prime}\right)\right\|<\hat{\gamma}^{-1}\left\|x-x^{\prime}\right\|+O(\varepsilon)\left\|x-x^{\prime}\right\| \leq\left(\hat{\gamma}^{-1}+O(\varepsilon)\right)\left\|x-x^{\prime}\right\| .
$$

In the same way one obtains that

$$
\left\|\psi(z, x)-\psi\left(z, x^{\prime}\right)\right\| \geq|\gamma-O(\varepsilon)|\left\|x-x^{\prime}\right\| .
$$

Taking $\varepsilon>0$ small enough we can assume $\hat{\gamma}^{-1}$ and $\gamma$ remain, respectively, the rates of contraction and expansion for $\psi(z, \cdot)$. This calculation shows that the derivatives of $\psi(z, \cdot)$ and $\phi(z, \cdot)$ are $O(\varepsilon)$-close. Moreover, since $Q(z, \cdot)$ is $C^{1}$-close to the constant function $x \mapsto z$, it follows that $\psi(z, \cdot)$ and $\phi(z, \cdot)$ are $O(\varepsilon)$-close. Consequently $d_{C^{1}}(\psi(z, \cdot), \phi(z, \cdot)) \leq O(\varepsilon)$.

Although for each $z \in \Lambda$ the maps $\psi(z, \cdot)$ are $C^{1}$-diffeomorphisms, the map $\tilde{g}$ is not necessarily a $C^{1}$-diffeomorphism since $h$ is not a $C^{1}$-conjugation. However, according to [IN12, Theorem A] it follows that both $h$ and $h^{-1}$ are locally $\alpha$-Hölder continuous maps with 
$\alpha=\log v / \log \mu>0$. Thus, the function $\psi=\pi_{M} \circ g \circ h^{-1}$ is locally $\alpha$-Hölder continuos with respect to the base points: if $d_{N}\left(z, z^{\prime}\right)<\delta$ it holds that

$$
d_{C^{0}}\left(\psi(z, \cdot), \psi\left(z^{\prime}, \cdot\right)\right) \leq L_{g} d_{C^{0}}\left(h^{-1}(z, \cdot), h^{-1}\left(z^{\prime}, \cdot\right)\right) \leq L_{g} C_{h} d_{N}\left(z, z^{\prime}\right)^{\alpha}
$$

where $L_{g}$ and $C_{h}$ are the local Lipschitz and $\alpha$-Hölder constants of $g$ and $h$ respectively.

Recall that $\left.F\right|_{\Lambda}$ is conjugated to the Bernoulli shift $\tau: \Sigma_{d} \rightarrow \Sigma_{d}$. Let $\ell: \Sigma_{d} \rightarrow \Lambda$ be the topological conjugation: $\tau=\left.\ell^{-1} \circ F\right|_{\Lambda} \circ \ell$. Consider $\Sigma_{d}$ with the metric given in (1.2), then from [Gor06, Theorem 2.3] and [BGMV03] it follows that $\ell$ is a Lipschitz map. That is $d_{M}\left(\ell(\xi), \ell\left(\xi^{\prime}\right)\right) \leq L_{\ell} d_{\Sigma_{d}}\left(\xi, \xi^{\prime}\right)$.

Therefore, we obtain that $\tilde{g}$ is conjugated to a locally $\alpha$-Hölder symbolic skew-product map

$$
\Psi_{g}: \Sigma_{d} \times M \rightarrow \Sigma_{d} \times M, \quad \Psi_{g}(\xi, x)=\left(\tau(\xi), \psi_{\xi}(x)\right),
$$

where $\psi_{\xi}=\psi(\ell(\xi), \cdot): M \rightarrow M$ is a $\left(\gamma, \hat{\gamma}^{-1}\right)$-Lipschitz $C^{1}$-diffeomorphism with the local Hölder constant $C_{\Psi_{g}}=L_{g} C_{h} L_{\ell}^{\alpha} \geq 0$ and $\alpha=\log v / \log \mu>0$.

The same arguments work for a small perturbation $g^{-1}$ of $f^{-1}$ and therefore the inverse map $\Psi_{g}^{-1}$ is also a skew-product with the same Hölder exponent $0<\alpha \leq 1$ and local Hölder constant $C_{\Psi} \geq 0$.

To end the proof we need to show that $C_{\Psi_{g}}=L_{f}+O(\varepsilon)$. To do this note that $L_{g}$ is the local Lipschitz constant of $g\left(\right.$ or $\left.g^{-1}\right)$ i.e.,

$$
L_{g}=\sup \left\{\frac{d\left(g(z, x), g\left(z^{\prime}, x^{\prime}\right)\right)}{d\left((z, x),\left(z^{\prime}, x^{\prime}\right)\right)}:(z, x),\left(z^{\prime}, x^{\prime}\right) \in \Delta, d_{N}\left(z^{\prime} z\right)<\delta\right\}
$$

and then $L_{g}=L_{f}+O(\varepsilon)$. Also, the local Hölder constant $C_{h}$ of $h\left(\right.$ or $\left.h^{-1}\right)$ varies continuosly with $h$ that in turn depends continuously on $g$. In fact, in view of (A.6) we get that $h$ and $h^{-1}$ are close to the identity and so $C_{h} L_{\ell}^{\alpha}=1+O(\varepsilon)$. Thus, $C_{\Psi_{g}}=L_{g} C_{h} L_{\ell}^{\alpha}=L_{f}+O(\varepsilon)$, which concludes the proof of the proposition.

Note that the above result also holds if $f$ is only defined locally as a skew-product diffeomorphism over the rectangles coming from a Markov partition of $\Lambda$. This fact is used to define locally constant skew-product diffeomorphisms in the proof of Theorem A and Theorem B.

\section{Appendix B. Proof of Theorem 2.3}

In this appendix we complete the details of the proof of Theorem 2.3.

Next result claims the existence of a "unique invariant attracting graph" on $\Sigma_{k} \times \bar{D}$ for $\Phi \in \mathcal{S}$. Indeed these graphs depend continuously on $\Phi$. The theorem below is a reformulation of the results in [HPS77] (see also [Sta99, Theorem 1.1] and [BHN99, Section 6] for Item (iii)).

Given a function $g: \Sigma_{k} \rightarrow \bar{D}$ its graph map is defined by

$$
\operatorname{graph}[g]: \Sigma_{k} \rightarrow \Sigma_{k} \times M, \quad \operatorname{graph}[g](\xi)=(\xi, g(\xi))
$$


and its graph set by $\Gamma_{g} \stackrel{\text { def }}{=}$ image $($ graph $[g])=\left\{(\xi, g(\xi)): \xi \in \Sigma_{k}\right\} \subset \Sigma_{k} \times \bar{D}$.

Theorem B.1 ([HPS77, Sta99, BHN99]). Consider $\Phi=\tau \ltimes \phi_{\xi} \in \mathcal{S}$ with $\beta<1$ and $\alpha \geq 0$. Then there exists a unique bounded continuous function $g_{\Phi}: \Sigma_{k} \rightarrow \bar{D}$ such that

i) $\Phi\left(\xi, g_{\Phi}(\xi)\right)=\left(\tau(\xi), g_{\Phi} \circ \tau(\xi)\right)$, for all $\xi \in \Sigma_{k}$, that is, $\Phi\left(\Gamma_{g_{\Phi}}\right)=\Gamma_{g_{\Phi^{\prime}}}$

ii) for every $(\xi, x) \in \Sigma_{k} \times \bar{D}$ and $n \geq 0$, it holds

$$
\left\|\phi_{\xi}^{n}(x)-g_{\Phi}\left(\tau^{n}(\xi)\right)\right\| \leq \beta^{n}\left\|g_{\Phi}(\xi)-x\right\|,
$$

iii) the map $\mathcal{L}: \mathcal{S} \rightarrow C^{0}\left(\Sigma_{k}, \bar{D}\right)$ given by $\mathcal{L}(\Phi)=g_{\Phi}$ is continuous.

For notational simplicity in what follows we just write $\Gamma_{\Phi}$ in place of $\Gamma_{g_{\Phi}}$ to denote the unique contracting invariant graph set. The next proposition shows that $\Gamma_{\Phi}$ is the locally maximal invariant set in $\Sigma_{k} \times \bar{D}$ and it is a consequence of previous theorem. This completes the first item in Theorem 2.3.

Proposition B.2. Consider $\Phi=\tau \ltimes \phi_{\xi} \in \mathcal{S}$ with $\beta<1$ and $\alpha \geq 0$. Then

i) the restriction $\left.\Phi\right|_{\Gamma_{\Phi}}$ of $\Phi$ to the set $\Gamma_{\Phi}$ is conjugate to $\tau$, and

ii) the graph set $\Gamma_{\Phi}$ is the (forward) maximal invariant set in $\Sigma_{k} \times \bar{D}$

$$
\Gamma_{\Phi}=\bigcap_{n \in \mathbb{Z}} \Phi^{n}\left(\Sigma_{k} \times \bar{D}\right)=\bigcap_{n \in \mathbb{N}} \Phi^{n}\left(\Sigma_{k} \times \bar{D}\right) .
$$

The following proposition is a local version of Proposition 2.1.

Proposition B.3. Consider $\Phi=\tau \ltimes \phi_{\xi} \in \mathcal{S}$ with $\beta<1, \alpha>0$, and let $\Gamma_{\Phi}$ be the maximal invariant set of $\Phi$ in $\Sigma_{k} \times \bar{D}$. Then, there exists a partition

$$
W_{\Gamma_{\Phi}}^{u}=\left\{W_{l o c}^{u u}((\xi, x) ; \Phi):(\xi, x) \in \Gamma_{\Phi}\right\}
$$

of $\Gamma_{\Phi}$ such that for every $(\xi, x) \in \Gamma_{\Phi}$ it holds

i) $W_{l o c}^{u u}((\xi, x) ; \Phi)$ is the graph of an $\alpha$-Hölder map $\gamma_{\xi}^{u}: W_{l o c}^{u}(\xi ; \tau) \rightarrow M$,

ii) $\gamma_{\xi}^{u}(\xi)=g_{\Phi}(\xi)=x$,

iii) $\gamma_{\xi}^{u}\left(\xi^{\prime \prime}\right)=\gamma_{\xi^{\prime}}^{u}\left(\xi^{\prime \prime}\right)$ for all $\xi^{\prime}, \xi^{\prime \prime} \in W_{l o c}^{u}(\xi ; \tau)$,

iv) $\phi_{\tau^{-1}\left(\xi^{\prime}\right)}^{-1} \circ \gamma_{\xi}^{u}\left(\xi^{\prime}\right)=\gamma_{\tau^{-1}(\xi)}^{u} \circ \tau^{-1}\left(\xi^{\prime}\right)$ where $\xi^{\prime} \in W_{l o c}^{u}(\xi ; \tau)$, and

v) $W_{l o c}^{u u}((\xi, x) ; \Phi) \subset W^{u}((\xi, x) ; \Phi)$.

Proof. Let $(\xi, x) \in \Gamma_{\Phi}$. Note that $x=g_{\Phi}(\xi)$ and that $\left(\phi_{\tau^{-n}(\xi)}^{n}\right)^{-1}=\phi_{\tau^{-1}(\xi)}^{-n}$ indeed:

$$
\phi_{\tau^{-n}(\xi)}^{-1} \circ \cdots \circ \phi_{\tau^{-1}(\xi)}^{-1} \circ \phi_{\tau^{-1}(\xi)} \circ \cdots \circ \phi_{\tau^{-n}(\xi)}=\phi_{\tau^{-1}(\xi)}^{-n} \circ \phi_{\tau^{-n}(\xi)}^{n}=i d .
$$

We define a sequence of maps $\gamma_{\xi}^{u, n}: W_{l o c}^{u}(\xi ; \tau) \rightarrow M$ by

$$
\gamma_{\xi}^{u, n}\left(\xi^{\prime}\right)=\phi_{\tau^{-n}\left(\xi^{\prime}\right)}^{n} \circ\left(\phi_{\tau^{-n}(\xi)}^{n}\right)^{-1}(x)=\phi_{\tau^{-n}\left(\xi^{\prime}\right)}^{n} \circ \phi_{\tau^{-1}(\xi)}^{-n} \circ g_{\Phi}(\xi) .
$$

Note that $\left\{\gamma_{\xi}^{u, n}\right\}$ is a sequence in the complete metric space $C^{0}\left(W_{l o c}^{u}(\xi ; \tau), M\right)$. 
Lemma B.4. The sequence $\left\{\gamma_{\xi}^{u, n}\right\}$ is Cauchy sequence and so it converges to some continuous map $\gamma_{\xi}^{u}: W_{l o c}^{u}(\xi ; \tau) \rightarrow M$

Proof. Since $\beta, v<1$, to prove the first part in the lemma it is enough to see that

$$
\left\|\gamma_{\xi}^{u, n+1}\left(\xi^{\prime}\right)-\gamma_{\xi}^{u, n}\left(\xi^{\prime}\right)\right\| \leq C_{\Phi}\left(\beta v^{\alpha}\right)^{n+1} d_{\Sigma_{k}}\left(\xi, \xi^{\prime}\right)^{\alpha} .
$$

To prove this inequality first recall notation in (1.4) and observe that Item (i) of Theorem B.1 implies that for every $n>0$ and $\xi \in \Sigma_{k}$

$$
\phi_{\xi}^{n} \circ g_{\Phi}(\xi)=g_{\Phi} \circ \tau^{n}(\xi) \text { and } \phi_{\tau^{-1}(\xi)}^{-n} \circ g_{\Phi}(\xi)=g_{\Phi} \circ \tau^{-n}(\xi) \text {. }
$$

Since $\phi_{\xi}(\bar{D}) \subset D$ for all $\xi \in \Sigma_{k}$, then for every $0<i \leq n$

$$
\phi_{\tau^{-n}\left(\xi^{\prime}\right)}^{n-i} \circ \phi_{\tau^{-1}(\xi)}^{-n} \circ g_{\Phi}(\xi)=\phi_{\tau^{-n}\left(\xi^{\prime}\right)}^{n-i} \circ g_{\Phi} \circ \tau^{-n}(\xi) \in \bar{D} .
$$

To prove the inequality in (B.1), observe that $\left\|\gamma_{\xi}^{u, n+1}\left(\xi^{\prime}\right)-\gamma_{\xi}^{u, n}\left(\xi^{\prime}\right)\right\|$ is equal to

$$
\begin{aligned}
& \| \phi_{\tau^{-n-1}\left(\xi^{\prime}\right)}^{n+1} \circ \phi_{\tau^{-1}(\xi)}^{-n-1} \circ g_{\Phi}(\xi)- \\
& \leq \phi_{\tau^{-n}\left(\xi^{\prime}\right)}^{n} \circ \phi_{\tau^{-1}(\xi)}^{-n} \circ g_{\Phi}(\xi) \| \\
& \leq \beta_{\tau^{-n-1}\left(\xi^{\prime}\right)}^{n} \circ \phi_{\tau^{-n-1}(\xi)}^{-1} \circ \phi_{\tau^{-1}(\xi)}^{-n} \circ g_{\Phi}(\xi)- \\
& \quad \phi_{\tau^{-n-1}\left(\xi^{\prime}\right)} \circ \phi_{\tau^{-n-1}\left(\xi^{\prime}\right)}^{-1} \circ \phi_{\tau^{-1}(\xi)}^{-n} \circ g_{\Phi}(\xi) \| \\
& \leq \beta^{n+1}\left\|\phi_{\tau^{-n-1}(\xi)}^{-1} \circ \phi_{\tau^{-1}(\xi)}^{-n} \circ g_{\Phi}(\xi)-\phi_{\tau^{-n-1}\left(\xi^{\prime}\right)}^{-} \circ \phi_{\tau^{-1}(\xi)}^{-n} \circ g_{\Phi}(\xi)\right\| .
\end{aligned}
$$

As $\phi_{\tau^{-1}(\xi)}^{-n} \circ g_{\Phi}(\xi) \in \bar{D}$, then local Hölder continuity implies

$$
\begin{aligned}
\left\|\gamma_{\xi}^{u, n+1}\left(\xi^{\prime}\right)-\gamma_{\xi}^{u, n}\left(\xi^{\prime}\right)\right\| & \leq \beta^{n+1} C_{\Phi} d_{\Sigma_{k}}\left(\tau^{-n-1}(\xi), \tau^{-n-1}\left(\xi^{\prime}\right)\right)^{\alpha} \\
& \leq C_{\Phi}\left(\beta \nu^{\alpha}\right)^{n+1} d_{\Sigma_{k}}\left(\xi, \xi^{\prime}\right)^{\alpha} .
\end{aligned}
$$

The proof of the lemma is now complete.

To prove that $\mathcal{W}_{\Gamma_{\Phi}}^{u}$ is a partition of $\Gamma_{\Phi}$ we need to show that $W_{l o c}^{u u}((\xi, x) ; \Phi)$ is contained in $\Gamma_{\Phi}$ for all $(\xi, x) \in \Gamma_{\Phi}$. To do this let

$$
\left(\xi^{\prime}, x^{\prime}\right)=\left(\xi^{\prime}, \gamma_{\xi}^{u}\left(\xi^{\prime}\right)\right) \in W_{l o c}^{u u}((\xi, x) ; \Phi) .
$$

From (B.2) and noting that $x=g_{\Phi}(\xi)$ we have that

$$
\left\|g_{\Phi}\left(\xi^{\prime}\right)-\gamma_{\xi}^{u}\left(\xi^{\prime}\right)\right\|=\lim _{n \rightarrow \infty}\left\|\phi_{\tau^{-n}\left(\xi^{\prime}\right)}^{n} \circ g_{\Phi} \circ \tau^{-n}\left(\xi^{\prime}\right)-\phi_{\tau^{-n}\left(\xi^{\prime}\right)}^{n} \circ g_{\Phi} \circ \tau^{-n}(\xi)\right\| .
$$

Since the maps $\phi_{\xi}$ are $\beta$-contractions and $g_{\Phi}: \Sigma_{k} \rightarrow \bar{D}$, if $K$ is an upper bound of the diameter of $\bar{D}$ we get that

$$
\left\|g_{\Phi}\left(\xi^{\prime}\right)-\gamma_{\xi}^{u}\left(\xi^{\prime}\right)\right\| \leq \lim _{n \rightarrow \infty} \beta^{n}\left\|g_{\Phi} \circ \tau^{-n}\left(\xi^{\prime}\right)-g_{\Phi} \circ \tau^{-n}(\xi)\right\| \leq \lim _{n \rightarrow \infty} K \beta^{n}=0 .
$$

Thus $g_{\Phi}\left(\xi^{\prime}\right)=\gamma_{\xi}^{u}\left(\xi^{\prime}\right)=x^{\prime}$ and so $\left(\xi^{\prime}, x^{\prime}\right) \in \Gamma_{\Phi}$. That is,

$$
W_{l o c}^{u u}((\xi, x) ; \Phi) \subset \Gamma_{\Phi} \quad \text { for all }(\xi, x) \in \Gamma_{\Phi} .
$$

Indeed, we have proved that $\gamma_{\xi}^{u}\left(\xi^{\prime}\right)=g_{\Phi}\left(\xi^{\prime}\right)$ for all $\xi^{\prime} \in W_{l o c}^{u}(\xi ; \tau)$, proving (ii). In particular, $\gamma_{\xi}^{u}\left(\xi^{\prime \prime}\right)=g_{\Phi}\left(\xi^{\prime \prime}\right)=\gamma_{\xi^{\prime}}^{u}\left(\xi^{\prime \prime}\right)$ for all $\xi^{\prime}, \xi^{\prime \prime} \in W_{l o c}^{u}(\xi ; \tau)$, which proves (iii). 
To prove that $\gamma_{\xi}^{u}$ is an $\alpha$-Hölder map (Item (i)) first note that by the triangle inequality

$$
\left\|\gamma_{\xi}^{u, n}\left(\xi^{\prime}\right)-x\right\|=\left\|\gamma_{\xi}^{u, n}\left(\xi^{\prime}\right)-\gamma_{\xi}^{u, n}(\xi)\right\| \leq \sum_{i=1}^{n} s_{i}\left(\xi^{\prime}\right)
$$

where $x=g_{\Phi}(\xi)$ and $s_{i}\left(\xi^{\prime}\right)$ is given by

$$
\begin{aligned}
\| \phi_{\tau^{-n+i}\left(\xi^{\prime}\right)}^{n-i} \circ \phi_{\tau^{-n-1+i}\left(\xi^{\prime}\right)} \circ & \phi_{\tau^{-n}(\xi)}^{i-1} \circ \phi_{\tau^{-1}(\xi)}^{-n}(x)- \\
& \phi_{\tau^{-n+i}\left(\xi^{\prime}\right)}^{n-i} \circ \phi_{\tau^{-n-1+i}(\xi)} \circ \phi_{\tau^{-n}(\xi)}^{i-1} \circ \phi_{\tau^{-1}(\xi)}^{-n}(x) \| .
\end{aligned}
$$

Claim B.5. $s_{i}\left(\xi^{\prime}\right) \leq C_{\Phi}\left(\beta v^{\alpha}\right)^{n-i} d_{\Sigma_{k}}\left(\xi, \xi^{\prime}\right)^{\alpha}$, for every $i \in\{1, \ldots, n\}$.

We postpone the proof of this claim. Taking $n \rightarrow \infty$ in (B.3) we get

$$
\left\|\gamma_{\xi}^{u}\left(\xi^{\prime}\right)-\gamma_{\xi}^{u}(\xi)\right\| \leq C_{\gamma} d_{\Sigma_{k}}\left(\xi^{\prime}, \xi\right)^{\alpha} \quad \text { for all } \xi^{\prime} \in W_{l o c}^{s}(\xi ; \tau),
$$

where $C_{\gamma}=C_{\Phi}\left(1-\beta v^{\alpha}\right)^{-1}$, showing $\gamma_{\xi}^{u}$ is $\alpha$-Hölder. Indeed for $\xi^{\prime}, \xi^{\prime \prime} \in W_{\text {loc }}^{u}(\xi ; \tau)$ observe that $\gamma_{\xi}^{u}\left(\xi^{\prime}\right)=\gamma_{\xi^{\prime}}^{u}\left(\xi^{\prime}\right)$ and $\gamma_{\xi}^{u}\left(\xi^{\prime \prime}\right)=\gamma_{\xi^{\prime}}^{u}\left(\xi^{\prime \prime}\right)$. Thus

$$
\left\|\gamma_{\xi}^{u}\left(\xi^{\prime}\right)-\gamma_{\xi}^{u}\left(\xi^{\prime \prime}\right)\right\|=\left\|\gamma_{\xi^{\prime}}^{u}\left(\xi^{\prime}\right)-\gamma_{\xi^{\prime}}^{u}\left(\xi^{\prime \prime}\right)\right\| \leq C_{\gamma} d_{\Sigma_{k}}\left(\xi^{\prime}, \xi^{\prime \prime}\right)^{\alpha} .
$$

Note that the Hölder constant obtained is uniform on $\xi$ and $x=g_{\Phi}(\xi)$.

Proof of Claim B.5. For every $i \in\{1, \ldots, n\}$, we have that

$$
\begin{aligned}
s_{i}\left(\xi^{\prime}\right)= & \| \phi_{\tau^{-n+i}\left(\xi^{\prime}\right)}^{n-i} \circ \phi_{\tau^{-n-1+i}\left(\xi^{\prime}\right)} \circ \phi_{\tau^{-n}(\xi)}^{i-1} \circ \phi_{\tau^{-1}(\xi)}^{-n}(x)- \\
& \phi_{\tau^{-n+i}\left(\xi^{\prime}\right)}^{n-i} \circ \phi_{\tau^{-n-1+i}(\xi)} \circ \phi_{\tau^{-n}(\xi)}^{i-1} \circ \phi_{\tau^{-1}(\xi)}^{-n}(x) \| \\
\leq & \beta^{n-i} \| \phi_{\tau^{-n-1+i}\left(\xi^{\prime}\right)} \circ \phi_{\tau^{-n}(\xi)}^{i-1} \circ \phi_{\tau^{-1}(\xi)}^{-n}(x)- \\
& \phi_{\tau^{-n-1+i}(\xi)} \circ \phi_{\tau^{-n}(\xi)}^{i-1} \circ \phi_{\tau^{-1}(\xi)}^{-n}(x) \| \\
\leq & \beta^{n-i} C_{\Phi} d_{\Sigma_{k}}\left(\tau^{-n-1+i}\left(\xi^{\prime}\right), \tau^{-n-1+i}(\xi)\right)^{\alpha} \\
\leq & \left(\beta v^{\alpha}\right)^{n-i} C_{\Phi} d_{\Sigma_{k}}\left(\xi^{\prime}, \xi\right)^{\alpha},
\end{aligned}
$$

where the first inequality follows from the $\beta$-contraction of $\phi_{\xi}$, and the second one from the $\alpha$-Hölder continuity of $\phi_{\xi}$.

This completes the proof of Item (i).

To prove Item (iv) observe that

$$
\begin{aligned}
\phi_{\tau^{-1}\left(\xi^{\prime}\right)}^{-1} \circ \gamma_{\xi}^{u}\left(\xi^{\prime}\right) & =\lim _{n \rightarrow \infty} \phi_{\tau^{-1}\left(\xi^{\prime}\right)}^{-1} \circ \phi_{\tau^{-n}\left(\xi^{\prime}\right)}^{n} \circ \phi_{\tau^{-1}(\xi)}^{-n} \circ g_{\Phi}(\xi) \\
& =\lim _{n \rightarrow \infty} \phi_{\tau^{-1}\left(\xi^{\prime}\right)}^{-1} \circ \phi_{\tau^{-1}\left(\xi^{\prime}\right)} \circ \phi_{\tau^{-n}\left(\xi^{\prime}\right)}^{n-1} \circ \phi_{\tau^{-1}(\xi)}^{-n} \circ g_{\Phi}(\xi) \\
& =\lim _{n \rightarrow \infty} \phi_{\tau^{-n}\left(\xi^{\prime}\right)}^{n-1} \circ \phi_{\tau^{-1}(\xi)}^{-n} \circ g_{\Phi}(\xi) \\
& =\lim _{n \rightarrow \infty} \phi_{\tau^{-n}\left(\xi^{\prime}\right)}^{n-1} \circ \phi_{\tau^{-2}(\xi)}^{-(n-1)} \circ \phi_{\tau^{-1}(\xi)}^{-1} \circ g_{\Phi}(\xi) \\
& =\lim _{n \rightarrow \infty} \phi_{\tau^{-n}\left(\xi^{\prime}\right)}^{n-1} \circ \phi_{\tau^{-2}(\xi)}^{-(n-1)} \circ g_{\Phi} \circ \tau^{-1}(\xi) \\
& =\gamma_{\tau^{-1}(\xi)}^{u} \circ \tau^{-1}\left(\xi^{\prime}\right),
\end{aligned}
$$


where equality (B.4) is consequence of (B.2): $\phi_{\tau^{-1}(\xi)}^{-1} \circ g_{\Phi}(\xi)=g_{\Phi} \circ \tau^{-1}(\xi)$.

Now we prove Item $(\mathrm{v}): W_{l o c}^{u u}((\xi, x) ; \Phi) \subset W^{u}((\xi, x) ; \Phi)$. Consider $\left(\xi^{\prime}, x^{\prime}\right) \in W_{l o c}^{u u}((\xi, x) ; \Phi)$. Then $\xi^{\prime} \in W_{l o c}^{u}(\xi ; \tau)$ and $x^{\prime}=\gamma_{\xi}^{u}\left(\xi^{\prime}\right)$. Thus,

$$
\begin{aligned}
& d\left(\Phi^{-n}\left(\xi^{\prime}, x^{\prime}\right), \Phi^{-n}(\xi, x)\right)= \\
& \quad=d_{\Sigma_{k}}\left(\tau^{-n}\left(\xi^{\prime}\right), \tau^{-n}(\xi)\right)+\left\|\phi_{\tau^{-1}\left(\xi^{\prime}\right)}^{-n}\left(x^{\prime}\right)-\phi_{\tau^{-1}(\xi)}^{-n}(x)\right\| \\
& \quad \leq v^{n} d_{\Sigma_{k}}\left(\xi^{\prime}, \xi\right)+\left\|\phi_{\tau^{-1}\left(\xi^{\prime}\right)}^{-n} \circ \gamma_{\xi}^{u}\left(\xi^{\prime}\right)-\phi_{\tau^{-1}(\xi)}^{-n}(x)\right\| .
\end{aligned}
$$

Since $(\xi, x) \in \Gamma_{\Phi}$ we have $x=g_{\Phi}(\xi)$, and using that $\gamma_{\tau^{-n}(\xi)}^{u} \circ \tau^{-n}(\xi)=g_{\Phi} \circ \tau^{-n}(\xi)=$ $\phi_{\tau^{-1}(\xi)}^{-n}(x)$ and $\phi_{\tau^{-1}\left(\xi^{\prime}\right)}^{-n} \circ \gamma_{\xi}^{u}\left(\xi^{\prime}\right)=\gamma_{\tau^{-n}(\xi)}^{u} \circ \tau^{-n}\left(\xi^{\prime}\right)$ we get

$$
\begin{aligned}
\left\|\phi_{\tau^{-1}\left(\xi^{\prime}\right)}^{-n} \circ \gamma_{\xi}^{u}\left(\xi^{\prime}\right)-\phi_{\tau^{-1}(\xi)}^{-n}(x)\right\|= \\
\quad=\left\|\gamma_{\tau^{-n}(\xi)}^{u} \circ \tau^{-n}\left(\xi^{\prime}\right)-\gamma_{\tau^{-n}(\xi)}^{u} \circ \tau^{-n}(\xi)\right\| \leq v^{\alpha n} d_{\Sigma_{k}}\left(\xi^{\prime}, \xi\right)^{\alpha} .
\end{aligned}
$$

This implies (B.5) goes to zero as $n$ goes to infinity and therefore $\left(\xi^{\prime}, x^{\prime}\right)$ belongs to $W^{u}((\xi, x) ; \Phi)$. The proof of Item (v) and of the proposition is now complete.

Recall that we denote by $\operatorname{Per}(\Phi)$ the set of periodic points of $\Phi$ and that $\mathscr{P}: \Sigma_{k} \times M \rightarrow M$ is the projection on the fiber space.

Proposition B.6. Consider $\Phi=\tau \ltimes \phi_{\xi} \in \mathcal{S}$ with $\beta<1, \alpha>0$. Then

i) $W^{u u}((\xi, x) ; \Phi)=W^{u}((\xi, x) ; \Phi) \subset \Gamma_{\Phi}$ for all $(\xi, x) \in \Gamma_{\Phi}$, and

ii) for all periodic point $(\vartheta, p)$ of $\Phi$ in $\Sigma_{k} \times \bar{D}$

$$
K_{\Phi} \stackrel{\text { def }}{=} \overline{\mathscr{P}(\operatorname{Per}(\Phi)) \cap D}=\overline{\mathscr{P}\left(W^{u}((\vartheta, p) ; \Phi)\right)}=\mathscr{P}\left(\Gamma_{\Phi}\right)=g_{\Phi}\left(\Sigma_{k}\right) .
$$

Proof. To prove the inclusion $W^{u u}((\xi, x) ; \Phi) \subset W^{u}((\xi, x) ; \Phi)$ for all $(\xi, x)$ in $\Gamma_{\Phi}$, we take $\left(\xi^{\prime}, x^{\prime}\right) \in$ $W^{u u}((\xi, x) ; \Phi)$ and show that

$$
\lim _{n \rightarrow \infty} d\left(\Phi^{-n}\left(\xi^{\prime}, x^{\prime}\right), \Phi^{-n}(\xi, x)\right)=0 .
$$

Since $\left(\xi^{\prime}, x^{\prime}\right) \in W^{u u}((\xi, x) ; \Phi)$, by definition there are $m \in \mathbb{N}$ and $(\zeta, z) \in W_{l o c}^{u u}\left(\Phi^{-m}(\xi, x) ; \Phi\right)$ such that $\left(\xi^{\prime}, x^{\prime}\right)=\Phi^{m}(\zeta, z)$. Let $(\eta, y)=\Phi^{-m}(\xi, x)$. Note that $(\eta, y) \in \Gamma_{\Phi},(\zeta, z) \in W_{l o c}^{u u}((\eta, y) ; \Phi)$, and

$$
d\left(\Phi^{-n}\left(\xi^{\prime}, x^{\prime}\right), \Phi^{-n}(\xi, x)\right)=d\left(\Phi^{-(n-m)}(\zeta, z), \Phi^{-(n-m)}(\eta, y)\right)
$$

By Item (v) of Proposition B.3, we have that $W_{l o c}^{u u}((\eta, y) ; \Phi) \subset W^{u}((\eta, y) ; \Phi)$ and thus it follows (B.6).

Let us prove the converse inclusion: if $(\xi, x) \in \Gamma_{\Phi}$ then $W^{u}((\xi, x) ; \Phi) \subset W^{u u}((\xi, x) ; \Phi)$. Take $\left(\xi^{\prime}, x^{\prime}\right) \in W^{u}((\xi, x) ; \Phi)$ and we have to show that there is $m \in \mathbb{N}$ such that $\Phi^{-m}\left(\xi^{\prime}, x^{\prime}\right) \in$ $W_{l o c}^{u u}\left(\Phi^{-m}(\xi, x) ; \Phi\right)$. By definition of the unstable set,

$$
\begin{gathered}
\lim _{n \rightarrow \infty} d_{\Sigma_{k}}\left(\tau^{-n}\left(\xi^{\prime}\right), \tau^{-n}(\xi)\right)=0 \quad \text { and } \\
\lim _{n \rightarrow \infty}\left\|\phi_{\tau^{-1}\left(\xi^{\prime}\right)}^{-n}\left(x^{\prime}\right)-\phi_{\tau^{-1}(\xi)}^{-n}(x)\right\|=0 .
\end{gathered}
$$


Since $(\xi, x) \in \Gamma_{\Phi}$ then $\phi_{\tau^{-1}(\xi)}^{-n}(x) \in D$ for all $n \geq 0$. Thus there exists $m \in \mathbb{N}$ such that

$$
\tau^{-m}\left(\xi^{\prime}\right) \in W_{l o c}^{u}\left(\tau^{-m}(\xi) ; \tau\right) \quad \text { and } \quad \phi_{\tau^{-1}\left(\xi^{\prime}\right)}^{-(n+m)}\left(x^{\prime}\right) \in D, \quad \text { for every } n \geq m
$$

Write $(\eta, y)=\Phi^{-m}(\xi, x) \in \Gamma_{\Phi}$ and $\left(\eta^{\prime}, y^{\prime}\right)=\Phi^{-m}\left(\xi^{\prime}, x^{\prime}\right)$. Hence $y=g_{\Phi}(\eta)$ and $\phi_{\tau^{-n}(\eta)}^{n-i} \circ \phi_{\tau^{-1}(\eta)}^{-n}(y)$, $\phi_{\tau^{-n}\left(\eta^{\prime}\right)}^{n-i} \circ \phi_{\tau^{-1}\left(\eta^{\prime}\right)}^{-n}\left(y^{\prime}\right)$ belong to $D$ for all $0<i \leq n$. Recalling the definition of $\gamma_{\eta}^{u}$ and $\gamma_{\eta}^{u, n}$ we have that

$$
\begin{aligned}
\left\|y^{\prime}-\gamma_{\eta}^{u}\left(\eta^{\prime}\right)\right\| & =\lim _{n \rightarrow \infty}\left\|\phi_{\tau^{-n}\left(\eta^{\prime}\right)}^{n} \circ \phi_{\tau^{-1}\left(\eta^{\prime}\right)}^{-n}\left(y^{\prime}\right)-\phi_{\tau^{-n}\left(\eta^{\prime}\right)}^{n} \circ \phi_{\tau^{-1}(\eta)}^{-n}(y)\right\| \\
& \leq \lim _{n \rightarrow \infty} \beta^{n}\left\|\phi_{\tau^{-1}\left(\eta^{\prime}\right)}^{-n}\left(y^{\prime}\right)-\phi_{\tau^{-1}(\eta)}^{-n}(y)\right\| .
\end{aligned}
$$

From (B.7) and since $\beta<1$, we get this limit is zero and so $y^{\prime}=\gamma_{\eta}^{u}\left(\eta^{\prime}\right)$. That is $\Phi^{-m}\left(\xi^{\prime}, x^{\prime}\right) \in$ $W_{l o c}^{u u}\left(\Phi^{-m}(\xi, x) ; \Phi\right)$ and therefore $\left(\xi^{\prime}, x^{\prime}\right) \in W^{u u}((\xi, x) ; \Phi)$, concluding our assertion.

Note, we proved that $\left.W^{u u}((\xi, x) ; \Phi)=W^{u}(\xi, x) ; \Phi\right)$ for all $(\xi, x) \in \Gamma_{\Phi}$. Then by Proposition B.3, $\left.W^{u}(\xi, x) ; \Phi\right) \subset \Gamma_{\Phi}$ ending the first item of the proposition.

To prove Item (ii), consider a periodic point $\vartheta \in \Sigma_{k}$ of $\tau$ and note that $W^{u}(\vartheta ; \tau)$ and $\operatorname{Per}(\tau)$ are both dense in $\Sigma_{k}$. Using the conjugation in Proposition B.2 and item (i), we get

$$
\overline{\operatorname{Per}\left(\left.\Phi\right|_{\Gamma_{\Phi}}\right)}=\Gamma_{\Phi}=\overline{W^{u}\left(\left(\vartheta, g_{\Phi}(\vartheta)\right) ; \Phi\right)}=\overline{W^{u u}\left(\left(\vartheta, g_{\Phi}(\vartheta)\right) ; \Phi\right)}
$$

If $(\vartheta, p) \in \Sigma_{k} \times \bar{D}$ is a periodic point of $\Phi$, from the assumption $\phi_{\xi}(\bar{D}) \subset D$, we have that $\Phi^{n}(\vartheta, p) \in \Sigma_{k} \times D$ for all $n \in \mathbb{Z}$. Moreover, since $g_{\Phi}$ is the unique invariant graph of $\Phi$ restricted to $\Sigma_{k} \times \bar{D}$, by Item (ii) in Theorem B.1 we get $p=g_{\Phi}(\vartheta)$. From this,

$$
\operatorname{Per}\left(\left.\Phi\right|_{\Gamma_{\Phi}}\right)=\operatorname{Per}\left(\left.\Phi\right|_{\Sigma_{k} \times \bar{D}}\right)=\operatorname{Per}(\Phi) \cap\left(\Sigma_{k} \times D\right)
$$

Recalling that $K_{\Phi}$ is the closure of projection by $\mathscr{P}$ of the periodic points of $\Phi$ in $D$ and since the projection $\mathscr{P}$ is a closed map and $\Sigma_{k}$ is a compact set, Equations (B.8) and (B.9) imply that

$$
\mathscr{P}\left(\Gamma_{\Phi}\right)=\overline{\mathscr{P}\left(W^{u}((\vartheta, p) ; \Phi)\right)}=\overline{\mathscr{P}\left(\operatorname{Per}\left(\left.\Phi\right|_{\Gamma_{\Phi}}\right)\right)}=\overline{\mathscr{P}(\operatorname{Per}(\Phi)) \cap D} \stackrel{\text { def }}{=} K_{\Phi} .
$$

Finally, by definition $\mathscr{P}\left(\Gamma_{\Phi}\right)=g_{\Phi}\left(\Sigma_{k}\right)$ and from the above equation $K_{\Phi}=g_{\Phi}\left(\Sigma_{k}\right)$, completing the proof.

Observe that Proposition B.3 and B.6 provide the second and third items in Theorem 2.3. Now we will show that $K_{\Phi}$ depends continuously with respect to $\Phi$. Consider the set $\mathcal{K}(\bar{D})$ whose elements are the compact subsets of $\bar{D}$ endowed with the Hausdorff metric

$$
d_{H}(A, B) \stackrel{\text { def }}{=} \sup \{d(a, B), d(b, A): a \in A, b \in B\}, \quad A, B \in \mathcal{K}(\bar{D}),
$$

obtaining a complete metric space.

Define the map $\mathscr{L}: \mathcal{S} \rightarrow \mathcal{K}(\bar{D})$ given by $\mathscr{L}(\Phi) \stackrel{\text { def }}{=} K_{\Phi}$.

Proposition B.7. Consider $\Phi=\tau \ltimes \phi_{\xi} \in \mathcal{S}$ with $\beta<1, \alpha \geq 0$. Then, for each $\varepsilon>0$ there is $\delta>0$ such that for every $\Psi=\tau \ltimes \psi_{\xi} \in \mathcal{S}$ with

$$
d_{C^{0}}\left(\phi_{\xi}, \psi_{\xi}\right)<\delta \quad \text { it holds that } d_{H}\left(K_{\Phi}, K_{\Psi}\right)=d_{H}(\mathscr{L}(\Phi), \mathscr{L}(\Psi))<\varepsilon .
$$


The above result follows from Proposition B.1 and the inequality

$$
d_{H}\left(K_{\Phi}, K_{\Psi}\right)=d_{H}\left(g_{\Phi}\left(\Sigma_{k}\right), g_{\Psi}\left(\Sigma_{k}\right)\right) \leq d_{C^{0}}\left(g_{\Phi}, g_{\Psi}\right) .
$$

This completes the proof of Theorem 2.3.

\section{AcKNowledgements}

We are grateful to Lorenzo J. Díaz for his assistance and review of preliminar versions of this paper and Enrique R. Pujals for helpful conversations and comments. The first two authors would also like to express a deep gratitude to their respective scientific advisors, J. Ángel Rodríguez and Lorenzo J. Díaz for the support.

During the preparation of this article the authors were supported by the following fellowships: P. G. Barrientos by FPU-grant AP2007-031035, MTM2011-22956 project (Spain) and CNPq post-doctoral fellowship (Brazil); Y. Ki by CNPq-Brazil doctoral fellowship and CNPq Pós doutorado Júnior 503802/2012-3; A. Raibekas by CNPq-Brazil doctoral and CAPESPNPD-Brazil post-doctoral fellowships.

We thank Instituto Nacional de Matemática Pura e Aplicada (IMPA)-Brazil for its hospitality.

\section{REFERENCES}

[AV10] A. Avila and M. Viana. Extremal Lyapunov exponents: an invariance principle and applications. Invent. Math., 181(1):115-189, 2010.

[BD96] C. Bonatti and Lorenzo J. Díaz. Persistent nonhyperbolic transitive diffeomorphisms. Ann. of Math. (2), 143(2):357-396, 1996.

[BD08] C. Bonatti and L. J. Díaz. Robust heterodimensional cycles and $C^{1}$-generic dynamics. J. Inst. Math. Jussieu, 7(3):469-525, 2008.

[BD12] C. Bonatti and L. J. Díaz. Abundance of $C^{1}$-homoclinic tangencies. Trans. Amer. Math. Soc., 264:5111-5148, 2012.

[BDV95] C. Bonatti, L. J. Díaz, and M. Viana. Discontinuity of the Hausdorff dimension of hyperbolic sets. C. R. Acad. Sci. Paris Sér. I Math., 320(6):713-718, 1995.

[BDV05] C. Bonatti, L. J. Díaz, and M. Viana. Dynamics beyond uniform hyperbolicity, volume 102 of Encyclopaedia of Mathematical Sciences. Springer-Verlag, Berlin, 2005.

[BGMV03] C. Bonatti, X. Gómez-Mont, and M. Viana. Généricité d'exposants de Lyapunov non-nuls pour des produits déterministes de matrices. Ann. Inst. H. Poincaré Anal. Non Linéaire, 20(4):579-624, 2003.

[BHN99] D. Broomhead, D. Hadjiloucas, and M. Nicol. Random and deterministic perturbation of a class of skew-product systems. Dynam. Stability Systems, 14(2):115-128, 1999.

[Bon04] C. Bonatti. The global dynamics of $C^{1}$-generic diffeomorphisms or flows. conference in Second Latin American Congress of Mathematicians, Cancún, México, 2004.

[CP10] S. Crovisier and E. R. Pujals. Essential hyperbolicity and homoclinic bifurcations: a dichotomy phenomenon/mechanism for diffeomorphisms. ArXiv e-prints, November 2010.

[FM01] Ernest Fontich and Pau Martín. Arnold diffusion in perturbations of analytic integrable Hamiltonian systems. Discrete Contin. Dynam. Systems, 7(1):61-84, 2001.

[Gor06] A. S. Gorodetskii. Regularity of central leaves of partially hyperbolic sets and applications. Izv. Ross. Akad. Nauk Ser. Mat., 70(6):19-44, 2006. 
[HN11] A. J. Homburg and M. Nassiri. Robust minimality of iterated function system with two generators. Preprint, 2011.

[Hom12] A. J. Homburg. Circle diffeomorphisms forced by expanding circle maps. Ergodic Theory and Dynamical Systems, 32:2011-2024, 2012.

[HPS77] M. W. Hirsch, C. C. Pugh, and M. Shub. Invariant manifolds. Lecture Notes in Mathematics, Vol. 583. Springer-Verlag, Berlin, 1977.

[Hut81] John E. Hutchinson. Fractals and self-similarity. Indiana Univ. Math. J., 30(5):713-747, 1981.

[IN12] Yu. Ilyashenko and A. Negut. Hölder properties of perturbed skew products and fubini regained. Nonlinearity, 25(8):2377, 2012.

[New70] S. E. Newhouse. Nondensity of axiom A(a) on S2. In Global Analysis (Proc. Sympos. Pure Math., Vol. XIV, Berkeley, Calif., 1968), pages 191-202. Amer. Math. Soc., Providence, R.I., 1970.

[NP12] M. Nassiri and E. R. Pujals. Robust transitivity in hamiltonian dynamics. Ann. Sci. Éc. Norm. Supér., 45(2), 2012.

[Pal00] J. Palis. A global view of dynamics and a conjecture on the denseness of finitude of attractors. Astérisque, (261):xiii-xiv, 335-347, 2000. Géométrie complexe et systèmes dynamiques (Orsay, 1995).

[PS00] E. R. Pujals and M. Sambarino. Homoclinic tangencies and hyperbolicity for surface diffeomorphisms. Ann. of Math. (2), 151(3):961-1023, 2000.

[PSW12] C. Pugh, M. Shub, and A. Wilkinson. Hölder foliations, revisited. Journal of Modern Dynamics, pages 79-120, December 2012.

[RHRHTU07] F. Rodriguez-Hertz, M. A. Rodriguez-Hertz, A. Tahzibi, and R. Ures. A criterion for ergodicity of non-uniformly hyperbolic diffeomorphisms. Electron. Res. Announc. Math. Sci., 14:74-81, 2007.

[Sta99] J. Stark. Regularity of invariant graphs for forced systems. Ergodic Theory Dynam. Systems, 19(1):155-199, 1999.

[Wil71] R. F. Williams. Composition of contractions. Bol. Soc. Brasil. Mat., 2(2):55-59, 1971.

Instituto de Matemática e Estatística, Universidade Federal Fluminense,

Rua Mário Santos Braga s/n - Campus Valonguinhos, Niterói, RJ, Brasil

E-mail address: barrientos@uniovi.es

Instituto de Ciências Exatas, Universidade Federal Fluninense,

Rua Desembargador Ellis Hermydio Figueira, 783, Bairro Aterrado, Volta Redonda, RJ, Brasil

E-mail address: yuriki@impa.br

Instituto de Matemática e Estatística, Universidade Federal Fluminense, Rua Mário Santos Braga s/N - Campus Valonguinhos, Niterói, RJ, Brasil

E-mail address: artem@impa.br 